\title{
ASYMPTOTIC BEHAVIOUR OF A CLASS OF DEGENERATE ELLIPTIC-PARABOLIC OPERATORS: A UNITARY APPROACH
}

\author{
FABio PAROnetto ${ }^{1}$
}

\begin{abstract}
We study the asymptotic behaviour of a sequence of strongly degenerate parabolic equations $\partial_{t}\left(r_{h} u\right)-\operatorname{div}\left(a_{h} \cdot D u\right)$ with $r_{h}(x, t) \geqslant 0, r_{h} \in L^{\infty}(\Omega \times(0, T))$. The main problem is the lack of compactness, by-passed via a regularity result. As particular cases, we obtain $G$-convergence for elliptic operators $\left(r_{h} \equiv 0\right), G$-convergence for parabolic operators $\left(r_{h} \equiv 1\right)$, singular perturbations of an elliptic operator $\left(a_{h} \equiv a\right.$ and $r_{h} \rightarrow r$, possibly $\left.r \equiv 0\right)$.
\end{abstract}

Mathematics Subject Classification. 35J15, 35K10, 35M10, 45J45.

Received May 26, 2005. Revised January 5, 2006 and February 17, 2006.

Published online July 20, 2007.

\section{INTRODUCTION}

In the papers $[5,13,14]$ De Giorgi and Spagnolo introduced $G$-convergence for a class of elliptic operators, precisely for a class of elliptic operators in divergence form defined by an elliptic and simmetric matrix with bounded coefficients. Tartar extended this convergence to the non-simmetric (and then non-linear) case (see, for instance, [16] and [17]).

Later in [3] Colombini and Spagnolo defined $G$-convergence for a class of parabolic operators in divergence form still defined by a simmetric matrix with bounded coefficients depending, in this case, also on time. Before introducing the aim of this paper we recall the definition of $G$-convergence in both cases, denoting the convergence by $E G$ in the elliptic case and by $P G$ in the parabolic one, as extended to non-simmetric operators by Tartar (for a book containing results about both $E G$ and $P G$ convergences we refer to [7]).

Consider $n \in \mathbf{N}$ fixed. Moreover, for $\lambda_{0} \leqslant \Lambda_{0}$ and $M$ positive real numbers, denote by $\mathcal{M}_{U}\left(\lambda_{0}, \Lambda_{0}, M\right)$, with $U$ open set of $\mathbf{R}^{k}, k \in \mathbf{N}$, the class of $n \times n$ matrices defined as follows:

$$
\begin{aligned}
& a=\left[a_{i j}(y)\right]_{i, j=1}^{n} \in L^{\infty}(U) \quad \text { such that } \\
& \lambda_{0}|\xi|^{2} \leqslant(a(y) \cdot \xi, \xi) \leqslant \Lambda_{0}|\xi|^{2} \\
& |(a(y) \cdot \xi, \eta)| \leqslant M(a(y) \cdot \xi, \xi)^{1 / 2}(a(y) \cdot \eta, \eta)^{1 / 2}
\end{aligned}
$$

for every $\xi, \eta \in \mathbf{R}^{n}$, for a.e. $y \in U$.

Keywords and phrases. G-convergence, PDE of mixed type, linear elliptic and parabolic equations.

${ }^{1}$ Dipartimento di Matematica "Ennio De Giorgi", Università del Salento, via per Arnesano, 73100 Lecce, Italy;

fabio.paronetto@unile.it

(c) EDP Sciences, SMAI 2007 
Definition 1.1. Let $\Omega$ be a bounded open set of $\mathbf{R}^{n}$ and $T>0$. Consider a sequence $\left(a_{h}\right)_{h} \subset \mathcal{M}_{\Omega}\left(\lambda_{0}, \Lambda_{0}, M\right)$, $a_{h}=a_{h}(x)$ (referring to (1), in this case $k=n$ ). Given $a=a(x) \in \mathcal{M}_{\Omega}\left(\lambda_{0}, \Lambda_{0}, M\right)$ we say that

$$
a_{h} \stackrel{E G}{\longrightarrow} a \quad \text { in } \Omega
$$

if for every $f \in H^{-1}(\Omega)$ it results that

$$
\begin{array}{ll}
u_{h} \rightarrow u & \text { in } L^{2}(\Omega) \\
a_{h} \cdot D u_{h} \rightarrow a \cdot D u & \text { in } L^{2}(\Omega)^{n} \text {-weak }
\end{array}
$$

where $u_{h}$ and $u$ denote respectively the solutions (see Def. 2.5 with $r \equiv 0$ ) of

$$
\left\{\begin{array} { l l } 
{ - \operatorname { d i v } ( a _ { h } \cdot D v ) = f } & { \text { in } \Omega } \\
{ v = 0 } & { \text { in } \partial \Omega }
\end{array} \quad \left\{\begin{array}{ll}
-\operatorname{div}(a \cdot D v)=f & \text { in } \Omega \\
v=0 & \text { in } \partial \Omega .
\end{array}\right.\right.
$$

For a sequence $\left(a_{h}\right)_{h} \subset \mathcal{M}_{\Omega \times(0, T)}\left(\lambda_{0}, \Lambda_{0}, M\right), a_{h}=a_{h}(x, t)$ (referring to (1), in this case $\left.k=n+1\right)$, and given $a=a(x, t) \in \mathcal{M}_{\Omega \times(0, T)}\left(\lambda_{0}, \Lambda_{0}, M\right)$ we say that

$$
a_{h} \stackrel{P G}{\longrightarrow} a \quad \text { in } \Omega \times(0, T)
$$

if for every $f \in L^{2}\left(0, T ; H^{-1}(\Omega)\right)$ and $\varphi \in L^{2}(\Omega)$ it results that

$$
\begin{array}{ll}
u_{h} \rightarrow u & \text { in } L^{2}\left(0, T, L^{2}(\Omega)\right) \\
a_{h} \cdot D u_{h} \rightarrow a \cdot D u & \text { in } L^{2}\left(0, T, L^{2}(\Omega)^{n}\right) \text {-weak }
\end{array}
$$

where $u_{h}$ and $u$ denote respectively the solutions (see Def. 2.5 with $r \equiv 1$ ) of

$$
\left\{\begin{array} { l l } 
{ \frac { \partial v } { \partial t } - \operatorname { d i v } ( a _ { h } \cdot D v ) = f } & { \text { in } \Omega \times ( 0 , T ) } \\
{ v = 0 } & { \text { in } \partial \Omega \times ( 0 , T ) } \\
{ v = \varphi } & { \text { in } \Omega \times \{ 0 \} }
\end{array} \quad \left\{\begin{array}{ll}
\frac{\partial v}{\partial t}-\operatorname{div}(a \cdot D v)=f & \text { in } \Omega \times(0, T) \\
v=0 & \text { in } \partial \Omega \times(0, T) \\
v=\varphi & \text { in } \Omega \times\{0\} .
\end{array}\right.\right.
$$

In [3] the authors studied the connection between $E G$ and $P G$ convergence: in particular they proved that if $\left(a_{h}\right)_{h} \in \mathcal{M}_{\Omega \times(0, T)}\left(\lambda_{0}, \Lambda_{0}, M\right)$ satisfies

$$
\lim _{\tau \rightarrow 0} \sup _{h} \int_{I} \int_{\omega}\left|a_{h}(x, t+\tau)-a_{h}(x, t)\right| \mathrm{d} x \mathrm{~d} t=0 \quad \forall I \times \omega \subset \subset(0, T) \times \Omega
$$

then

$$
a_{h}(\cdot, t) \stackrel{E G}{\longrightarrow} a(\cdot, t) \quad \text { in } \Omega \text { for a.e. } t \in(0, T) \quad \text { iff } \quad a_{h} \stackrel{P G}{\longrightarrow} a \quad \text { in } \Omega \times(0, T)
$$

and showed with a counterexample that this is not always true.

In this paper we consider strongly degenerate parabolic, or elliptic-parabolic, operators like

$$
P u=\frac{\partial}{\partial t}(r u)-\operatorname{div}(a \cdot D u) \quad \text { with } r=r(x, t) \geqslant 0
$$


and study the limit behaviour of the sequence of Cauchy-Dirichlet problems (for the existence result we refer to $[8]$, but see also [11])

$$
\begin{cases}\frac{\partial}{\partial t}\left(r_{h} u\right)-\operatorname{div}\left(a_{h} \cdot D u\right)=f & \text { in } \Omega_{h,+}(t) \times(0, T) \\ -\operatorname{div}\left(a_{h} \cdot D u\right)=f & \text { in } \Omega_{h, 0}(t) \times(0, T) \\ u=0 & \text { in } \partial \Omega \times(0, T) \\ u=\varphi & \text { in } \Omega_{h,+}(0) \times\{0\}\end{cases}
$$

where (the initial condition on $\Omega_{h,+}(0)$ will be clarified at the end of Sect. 2) $\Omega_{h,+}(t):=\left\{x \in \Omega \mid r_{h}(x, t)>0\right\}$ and $\Omega_{h, 0}(t):=\left\{x \in \Omega \mid r_{h}(x, t)=0\right\},\left(a_{h}\right)_{h}$ is a sequence in $\mathcal{M}_{\Omega \times(0, T)}\left(\lambda_{0}, \Lambda_{0}, M, N\right)$, the class of matrices $a$ satisfying (1) and

$$
|a(x, t)-a(x, s)| \leqslant N|t-s|
$$

for a.e. $x \in \Omega$ and every $s, t \in[0, T], r_{h}$ belonging to a suitable class $\mathcal{F}$ defined in (6). Arising from (3) the aim of the present paper is to give a more general definition of $G$-convergence, for problems (5) (see Def. 4.3), which is independent of the sequence $\left(r_{h}\right)_{h}$ and a compactness result with respect to it (see Th. 4.5). This in particular justifies (3) and includes other phenomena, as singular perturbations (in which the result is stronger, see Prop. 5.1), but we refer to the lastgli Studi di Lecce section for examples. We want to stress that, since $r$ in (4) may be equal to zero on some region with positive measure, a difficulty in this situation is that the natural compactness result (see Th. 2.7) is not guaranteed. Only for the sequence of the solutions (the solutions $u_{h}$ to the problems $(5)$ ), we are able to obtain the compactness via a regularity result (see Th. 2.8).

We recall that, in the general situation, a first study in this direction was already made, in the periodic case and with $r=r(x)$, in [9].

Elliptic-parabolic operators like those in (4) were already studied, as regards the existence of the solution, probably first by Showalter (see, for instance, [10] for one of the first papers and [11] for a recent book) and recently in [8] for a more general class of operators (nonlinear and possibly forward, backward and stationary).

The interest to study such problems lies on the fact that many diffusion problems lead to differential equations like

$$
\frac{\partial}{\partial t}(r(x, t) u)-\operatorname{div}(a(x, t) \cdot D u)=f
$$

which may be also of mixed type (see for example [1], Chap. 3, and the references therein), i.e. partially elliptic and partially parabolic. For some applications see also the examples in the last section.

The scheme of the paper is the following: Section 2 is dedicated to existence of the solution to an equation $P u=f$ and to the position of the problem. In Section 3 there are some compactness results: since a "classical type" compactness result (see Th. 2.7, with $r_{h} \equiv 1$ for the classical case) does not hold in general, we pass through a regularity result (Th. 2.8) to obtain it. In Section 4 we define $G$-convergence and prove a compactness result in two steps: Theorem 4.1 furnishes, given a sequence of operators $P_{h} u=\frac{\partial}{\partial t}\left(r_{h} u\right)-\operatorname{div}\left(a_{h} \cdot D u\right)$ in a suitable class, the existence, up to a subsequence, of a limit operator $P u=\frac{\partial}{\partial t}(r u)-\operatorname{div}\left(a_{r} \cdot D u\right)$, Theorem 4.5 states that $a_{r}$ is independent of $r$. In the last section we give some examples, including also singular perturbations.

\section{Elliptic-PARABOlic EQUATions AND STATEMENT OF THE PROBlEM}

From now on $T, \lambda_{0}, \Lambda_{0}, M$ will be fixed positive constants, $N, C_{1}, C_{2}$ non-negative constants, $\mu_{0}$ a non-positive constant (indeed $\mu_{0}$ could also be positive, but in that case it is sufficient to consider $\mu_{0}=0$ ) and $\Omega$ a bounded open set of $\mathbf{R}^{n}$ with Lipschitzian boundary. We will denote for brevity

$$
\mathcal{V}:=L^{2}\left(0, T ; H_{0}^{1}(\Omega)\right), \quad \mathcal{H}:=L^{2}\left(0, T ; L^{2}(\Omega)\right), \quad \mathcal{V}^{\prime}:=L^{2}\left(0, T ; H^{-1}(\Omega)\right)
$$


We denote by $\mathcal{F}\left(C_{1}, C_{2}, \mu_{0}\right)$ the class of measurable functions $r$ satisfying

$$
\begin{aligned}
& \text { (i) } r \in L^{\infty}(\Omega \times(0, T)), \quad r \geqslant 0, \\
& \text { (ii) }\|r\|_{L^{\infty}(\Omega \times(0, T))} \leqslant C_{1}, \\
& \text { (iii) } t \mapsto \int_{\Omega} u(x) v(x) r(x, t) \mathrm{d} x \quad \text { absolutely continuous on }[0, T], \\
& \text { (iv) }\left|\frac{\mathrm{d}}{\mathrm{d} t} \int_{\Omega} u(x) v(x) r(x, t) \mathrm{d} x\right| \leqslant C_{2}\|u\|_{H_{0}^{1}(\Omega)}\|v\|_{H_{0}^{1}(\Omega)} \quad \text { for a.e. } t \in[0, T], \\
& \text { (v) } \frac{\mathrm{d}}{\mathrm{d} t} \int_{\Omega} u^{2}(x) r(x, t) \mathrm{d} x \geqslant \mu_{0}\|u\|_{H_{0}^{1}(\Omega)}^{2} \quad \text { for a.e. } t \in[0, T]
\end{aligned}
$$

for every $u, v \in H_{0}^{1}(\Omega)$. For a $r \in \mathcal{F}\left(C_{1}, C_{2}, \mu_{0}\right)$ we define

$$
\Omega_{+}^{r}(t):=\{x \in \Omega \mid r(x, t)>0\}, \quad \Omega_{0}^{r}(t):=\{x \in \Omega \mid r(x, t)=0\} .
$$

Remark 2.1. The class just defined is compact, i.e. if $\left(r_{h}\right)_{h}$ is a sequence in $\mathcal{F}\left(C_{1}, C_{2}, \mu_{0}\right)$, there is a subsequence $\left(r_{h_{j}}\right)_{j}$ and a function $r \in \mathcal{F}\left(C_{1}, C_{2}, \mu_{0}\right)$ such that $r_{h_{j}} \rightarrow r$ in $L^{\infty}(\Omega \times(0, T))$-weak*. In fact, there is a subsequence $\left(r_{h_{j}}\right)_{j}$ and a function $r$ such that $r_{h_{j}} \rightarrow r$ in $L^{\infty}(\Omega \times(0, T))$-weak*. Now verify that $r$ belongs to $\mathcal{F}\left(C_{1}, C_{2}, \mu_{0}\right)$. Clearly $r \geqslant 0$ and $\|r\|_{\infty} \leqslant C_{1}$. To verify that $r$ satisfies also (iii), (iv), (v), consider a countable set $Z$, dense in $H_{0}^{1}(\Omega)$, and for every $u, v \in Z$ define the functions $F_{h}^{u, v}(t)=\int_{\Omega} u(x) v(x) r_{h}(x, t) \mathrm{d} x$. Since $\left(r_{h}\right)_{h} \subset \mathcal{F}\left(C_{1}, C_{2}, \mu_{0}\right)$ the sequence $\left(F_{h}^{u, v}\right)_{h}$ turns out to be equicontinuous and equibounded. Then there is a function, denoted by $F^{u, v}$, and a subsequence $F_{h_{j}}^{u, v}$ such that $F_{h_{j}}^{u, v} \rightarrow F^{u, v}$ uniformly in $[0, T]$. Since $Z$ is countable we can find a sequence $h_{j_{k}}$ such that $F_{h_{j_{k}}}^{u, v} \rightarrow F^{u, v}$ uniformly in $[0, T]$ for every $u, v \in Z$ (and in fact for every $\left.u, v \in H_{0}^{1}(\Omega)\right)$. This in particular implies that

$$
\int_{0}^{T} F_{h_{j_{k}}}^{u, v}(t) \eta(t) \mathrm{d} t \rightarrow \int_{0}^{T} F^{u, v}(t) \eta(t) \mathrm{d} t
$$

for every $\eta \in L^{1}(\Omega)$. Since $r_{h_{j}} \rightarrow r$ in $L^{\infty}(\Omega \times(0, T))$-weak*

$$
\int_{0}^{T} F_{h_{j_{k}}}^{u, v}(t) \eta(t) \mathrm{d} t=\int_{0}^{T} \int_{\Omega} u(x) v(x) \eta(t) r_{h_{j_{k}}}(x, t) \mathrm{d} x \mathrm{~d} t \rightarrow \int_{0}^{T} \int_{\Omega} u(x) v(x) \eta(t) r(x, t) \mathrm{d} x \mathrm{~d} t
$$

and then $F^{u, v}(t)=\int_{\Omega} u(x) v(x) r(x, t) \mathrm{d} x$.

Notice that $F^{u, v} \in W^{1, \infty}$ : then for every $\eta \in C_{c}^{1}(0, T)$ we have

$$
\int_{0}^{T} \frac{\mathrm{d}}{\mathrm{d} t}\left[F_{h_{j_{k}}}^{u, v}(t)\right] \eta(t) \mathrm{d} t=-\int_{0}^{T} F_{h_{j_{k}}}^{u, v}(t) \eta^{\prime}(t) \mathrm{d} t \rightarrow \int_{0}^{T} \frac{\mathrm{d}}{\mathrm{d} t}\left[F^{u, v}(t)\right] \eta(t) \mathrm{d} t
$$

and then we derive

$$
-C_{2}\|u\|_{H_{0}^{1}(\Omega)}\|v\|_{H_{0}^{1}(\Omega)} \int_{0}^{T} \eta(t) \mathrm{d} t \leqslant \int_{0}^{T} \frac{\mathrm{d}}{\mathrm{d} t}\left[F^{u, v}(t)\right] \eta(t) \mathrm{d} t \leqslant C_{2}\|u\|_{H_{0}^{1}(\Omega)}\|v\|_{H_{0}^{1}(\Omega)} \int_{0}^{T} \eta(t) \mathrm{d} t
$$

from which

$$
\left|\frac{\mathrm{d}}{\mathrm{d} t}\left[F^{u, v}(t)\right]\right| \leqslant C_{2}\|u\|_{H_{0}^{1}(\Omega)}\|v\|_{H_{0}^{1}(\Omega)} .
$$

Analogously we derive that $\frac{\mathrm{d}}{\mathrm{d} t} \int_{\Omega} u^{2}(x) r(x, t) \mathrm{d} x \geqslant \mu_{0}\|u\|_{H_{0}^{1}(\Omega)}^{2}$. 
Given $r \in \mathcal{F}\left(C_{1}, C_{2}, \mu_{0}\right)$ we introduce the families of operators

$$
\begin{aligned}
& R:[0, T] \longrightarrow \mathcal{L}\left(L^{2}(\Omega)\right), \quad R(t) u:=r(\cdot, t) u(\cdot) \\
& R^{\prime}:[0, T] \longrightarrow \mathcal{L}\left(H_{0}^{1}(\Omega), H^{-1}(\Omega)\right), \quad\left\langle R^{\prime}(t) u, v\right\rangle:=\frac{\mathrm{d}}{\mathrm{d} t} \int_{\Omega} u(x) v(x) r(x, t) \mathrm{d} x \\
& \mathcal{R}: \mathcal{H} \longrightarrow \mathcal{H} \quad \mathcal{R} u(t):=R(t) u(t) \\
& \mathcal{R}^{\prime}: \mathcal{V} \longrightarrow \mathcal{V}^{\prime} \quad\left\langle\mathcal{R}^{\prime} u, v\right\rangle_{\mathcal{V}^{\prime} \times \mathcal{V}}:=\int_{0}^{T}\left\langle R^{\prime}(t) u(t), v(t)\right\rangle_{H^{-1}(\Omega) \times H_{0}^{1}(\Omega)} \mathrm{d} t
\end{aligned}
$$

and define the following Banach space

$$
\mathcal{W}=\left\{u \in \mathcal{V} \mid(\mathcal{R} u)^{\prime} \in \mathcal{V}^{\prime}\right\}, \quad\|u\|_{\mathcal{W}}:=\|u\|_{\mathcal{V}}+\left\|(\mathcal{R} u)^{\prime}\right\|_{\mathcal{V}^{\prime}}
$$

where $(\mathcal{R} u)^{\prime}$ denotes the derivative in the distributional sense of $\mathcal{R} u$ with respect to the variable $t$.

An approximation result we will need later is the following.

Lemma 2.2. Consider $R$ defined in (8). Then for every $u \in \mathcal{W}$ and $\sigma>0$ there exist $v \in C^{1}\left([0, T] ; H_{0}^{1}(\Omega)\right)$ and $S \in C^{2}\left([0, T] ; \mathcal{L}\left(H_{0}^{1}(\Omega), H^{-1}(\Omega)\right)\right.$ ) (defined analogously to $R$ by a $s \in \mathcal{F}\left(C_{1}, C_{2}, \mu_{0}\right), \frac{\partial s}{\partial t} \in \mathcal{F}\left(C_{2}, K,-K\right)$ where $\left.K=K\left(C_{1}, \sigma\right)\right)$ such that

$$
\|u-v\|_{\mathcal{W}}<\sigma, \quad\left\|(\mathcal{R} u)^{\prime}-(\mathcal{S} v)^{\prime}\right\|_{\mathcal{V}^{\prime}}<\sigma
$$

Moreover $\mathcal{S}$ can be chosen in such a way that $S^{\prime}(0)=0$.

Proof. Fix $u \in \mathcal{W}=\mathcal{W}_{\mathcal{R}}$ and $\sigma>0$. From Proposition 2.4 in [8] we derive the existence of $v \in C^{1}\left([0, T] ; H_{0}^{1}(\Omega)\right)$ such that

$$
\|u-v\|_{\mathcal{W}}<\sigma / 2
$$

Consider a family of mollifiers $\left(\rho_{\epsilon}\right)_{\epsilon>0}$ and, after defining

$$
\bar{R}(t):= \begin{cases}R(t) & \text { if } t \in[0, T] \\ 0 & \text { if } t \notin[0, T]\end{cases}
$$

consider

$$
R_{\epsilon}(t)=\int_{\mathbf{R}} \bar{R}(\tau) \rho_{\epsilon}(t-\tau) \mathrm{d} \tau
$$

and the corresponding $\mathcal{R}_{\epsilon} u(t):=R_{\epsilon}(t) u(t)$. Note that $R \in W^{1, \infty}\left(0, T ; \mathcal{L}\left(H_{0}^{1}(\Omega), H^{-1}(\Omega)\right)\right)$ and $R_{\epsilon} \rightarrow R$ in $L^{\infty}\left(0, T ; \mathcal{L}\left(H_{0}^{1}(\Omega), H^{-1}(\Omega)\right)\right) \cap W^{1, q}\left(0, T ; \mathcal{L}\left(H_{0}^{1}(\Omega), H^{-1}(\Omega)\right)\right)$ for every $q<+\infty$.

Clearly $\left\langle R_{\epsilon}^{\prime}(t) u, u\right\rangle_{H^{-1}(\Omega) \times H_{0}^{1}(\Omega)}=\left\langle\int_{\mathbf{R}} \bar{R}^{\prime}(\tau) \rho_{\epsilon}(t-\tau) \mathrm{d} \tau u, u\right\rangle \geqslant \mu_{0}\|u\|_{H_{0}^{1}(\Omega)}^{2}$.

Observe that, since $v \in C^{1}\left([0, T] ; H_{0}^{1}(\Omega)\right), v \in \mathcal{W}_{\mathcal{R}}$ and $v \in \mathcal{W}_{\mathcal{R}_{\epsilon}}$ for every $\epsilon>0$. Then, since

$$
\left\|(\mathcal{R} u)^{\prime}-\left(\mathcal{R}_{\epsilon} v\right)^{\prime}\right\|_{\mathcal{V}^{\prime}} \leqslant\left\|(\mathcal{R} u)^{\prime}-(\mathcal{R} v)^{\prime}\right\|_{\mathcal{V}^{\prime}}+\left\|(\mathcal{R} v)^{\prime}-\left(\mathcal{R}_{\epsilon} v\right)^{\prime}\right\|_{\mathcal{V}^{\prime}}
$$

to prove the result it is sufficient to estimate $\left\|(\mathcal{R} v)^{\prime}-\left(\mathcal{R}_{\epsilon} v\right)^{\prime}\right\|_{\mathcal{V}^{\prime}}$. Since $R_{\epsilon} \rightarrow R$ in $L^{\infty}\left(0, T ; \mathcal{L}\left(H_{0}^{1}(\Omega), H^{-1}(\Omega)\right)\right)$ we have that

$$
\mathcal{R}_{\epsilon} v^{\prime} \rightarrow \mathcal{R} v^{\prime} \quad \text { in } \mathcal{H}
$$

and therefore it is sufficient to analyse the quantity $\mathcal{R}_{\epsilon}^{\prime} v-\mathcal{R}^{\prime} v$. We consider a function $\phi \in \mathcal{V}$ and by the 
generalized Hölder's inequality we have for every $p>2$

$$
\begin{aligned}
& \left|\left\langle\mathcal{R}_{\epsilon}^{\prime} v-\mathcal{R}^{\prime} v, \phi\right\rangle_{\mathcal{V}^{\prime} \times \mathcal{V}}\right|=\left|\int_{0}^{T}\left\langle R_{\epsilon}^{\prime}(t) v(t)-R^{\prime}(t) v(t), \phi(t)\right\rangle_{H^{-1} \times H_{0}^{1}} \mathrm{~d} t\right| \\
& \leqslant\left[\int_{0}^{T}\left\|R_{\epsilon}^{\prime}(t)-R^{\prime}(t)\right\|_{\mathcal{L}\left(H_{0}^{1}, H^{-1}\right)}^{2 p} \mathrm{~d} t\right]^{\frac{p-2}{2 p}}\left[\int_{0}^{T}\|v(t)\|_{H_{0}^{1}(\Omega)}^{p} \mathrm{~d} t\right]^{\frac{1}{p}}\left[\int_{0}^{T}\|\phi(t)\|_{H_{0}^{1}(\Omega)}^{2} \mathrm{~d} t\right]^{\frac{1}{2}} .
\end{aligned}
$$

Taking the supremum with respect to $\phi,\|\phi\|_{H_{0}^{1}(\Omega)}=1$, we obtain that

$$
\left\|\mathcal{R}_{\epsilon}^{\prime} v-\mathcal{R}^{\prime} v\right\|_{\mathcal{V}^{\prime}} \leqslant\left\|R_{\epsilon}^{\prime}-R^{\prime}\right\|_{L^{2 p /(p-2)}\left(0, T ; \mathcal{L}\left(H_{0}^{1}(\Omega), H^{-1}(\Omega)\right)\right)}\|v\|_{L^{p}\left(0, T ; H_{0}^{1}(\Omega)\right)}
$$

for a $p>2$ and since $R_{\epsilon}^{\prime} \rightarrow R^{\prime}$ in $L^{q}\left(0, T ; \mathcal{L}\left(H_{0}^{1}(\Omega), H^{-1}(\Omega)\right)\right)$ for every $q<+\infty$ we conclude choosing $\mathcal{S}=\mathcal{R}_{\epsilon}$ for $\epsilon$ sufficiently small.

Moreover we can choose $\mathcal{S}$ in such a way $S^{\prime}(0)=0$. To do this it is sufficient to consider the function $\eta(t)=\delta^{-1} t$ in $[0, \delta]$ and $\eta(t)=1$ in $[\delta, T]$, and choose

$$
S(t)=\int_{0}^{t} \eta(\tau) R_{\epsilon}^{\prime}(\tau) \mathrm{d} \tau+R_{\epsilon}(0)
$$

It is sufficient to estimate $\left\|\left(\mathcal{R}_{\epsilon} v\right)^{\prime}-(\mathcal{S} v)^{\prime}\right\|_{\mathcal{V}^{\prime}}$. First we estimate $\mathcal{R}_{\epsilon} v^{\prime}-\mathcal{S} v^{\prime}$ in $\mathcal{H}$ :

$$
\int_{0}^{T}\left\|R_{\epsilon}(t) v^{\prime}(t)-S(t) v^{\prime}(t)\right\|_{L^{2}(\Omega)}^{2} \mathrm{~d} t \leqslant\left\|R_{\epsilon}^{\prime}\right\|_{L^{\infty}\left(0, T ; \mathcal{L}\left(H_{0}^{1}(\Omega), H^{-1}(\Omega)\right)\right)}^{2}\left\|v^{\prime}\right\|_{\mathcal{V}}^{2} \delta^{2} .
$$

Similarly we can obtain $\left\|\mathcal{R}_{\epsilon}^{\prime} v-\mathcal{S}^{\prime} v\right\|_{\mathcal{V}^{\prime}} \leqslant\left\|R_{\epsilon}^{\prime}\right\|_{L^{\infty}\left(0, T ; \mathcal{L}\left(H_{0}^{1}(\Omega), H^{-1}(\Omega)\right)\right)}\|v\|_{\mathcal{V}} \sqrt{\delta}$. Since $\delta$ is arbitrary we are done.

For the following result see Proposition 2.6 in [8].

Theorem 2.3. For every $u, v \in \mathcal{W}$ the following holds:

$$
\begin{aligned}
& \frac{\mathrm{d}}{\mathrm{d} t}(\mathcal{R} u(t), v(t))_{L^{2}(\Omega)}=\left\langle\mathcal{R}^{\prime} u(t), v(t)\right\rangle_{H^{-1}(\Omega) \times H_{0}^{1}(\Omega)} \\
& \quad+\left\langle\mathcal{R} u^{\prime}(t), v(t)\right\rangle_{H^{-1}(\Omega) \times H_{0}^{1}(\Omega)}+\left\langle\mathcal{R} v^{\prime}(t), u(t)\right\rangle_{H^{-1}(\Omega) \times H_{0}^{1}(\Omega)}
\end{aligned}
$$

Moreover the function $t \mapsto(R(t) u(t), u(t))_{L^{2}(\Omega)}$ is continuous and there is a constant $c=c(T,\|\mathcal{R}\|)($ depending only on $\left.T,\|\mathcal{R}\| \leqslant C_{1}\right)$ such that

$$
\max _{[0, T]}\left|(R(t) u(t), u(t))_{L^{2}(\Omega)}\right| \leqslant c\|u\|_{\mathcal{W}}^{2} .
$$

Remark 2.4. Observe that, if $R(t) v(x)=r(x, t) v(x)$ for every $v \in L^{2}(\Omega)$ and for a $r \in \mathcal{F}\left(C_{1}, C_{2}, \mu_{0}\right)$, by Theorem 2.3 we deduce that if $u \in \mathcal{W}$ then

$$
u(t) \in L^{2}\left(\Omega_{+}^{r}(t), r(\cdot, t)\right) \quad \text { for every } t \in[0, T]
$$

where $\Omega_{+}^{r}(t)$ is defined in $(7)$ and $L^{2}\left(\Omega_{+}^{r}(t), r(\cdot, t)\right)$ denotes the completion of $L^{2}\left(\Omega_{+}^{r}(t)\right)$ with respect to the norm $\|v\|^{2}:=\int_{\Omega} v^{2}(x) r(x, t) \mathrm{d} x$. Observe that

$$
L^{2}(\Omega) \subset L^{2}\left(\Omega_{+}^{r}(t), r(\cdot, t)\right) \text { and } \int_{\Omega_{+}^{r}(t)} v^{2}(x) r(x, t) \mathrm{d} x \leqslant C_{1} \int_{\Omega} v^{2}(x) \mathrm{d} x
$$

for every $v \in L^{2}(\Omega)$ and every $t \in[0, T]$. 
We recall the definition of the class $\mathcal{M}_{\Omega \times(0, T)}\left(\lambda_{0}, \Lambda_{0}, M\right)$, with $\lambda_{0} \leqslant \Lambda_{0}$ and $M$ positive real numbers, given in (1), characterised by

$$
\begin{aligned}
& a=\left[a_{i j}(x, t)\right]_{i, j=1}^{n} \quad \text { such that } \\
& \lambda_{0}|\xi|^{2} \leqslant(a(x, t) \cdot \xi, \xi) \leqslant \Lambda_{0}|\xi|^{2} \\
& |(a(x, t) \cdot \xi, \eta)| \leqslant M(a(x, t) \cdot \xi, \xi)^{1 / 2}(a(x, t) \cdot \eta, \eta)^{1 / 2}
\end{aligned}
$$

for every $\xi, \eta \in \mathbf{R}^{n}$, for a.e. $(x, t) \in \Omega \times(0, T)$. By $\mathcal{M}_{\Omega \times(0, T)}\left(\lambda_{0}, \Lambda_{0}, M, N\right), N$ non-negative constant, we denote the subclass of $\mathcal{M}_{\Omega \times(0, T)}\left(\lambda_{0}, \Lambda_{0}, M\right)$ satisfying the further assumption

$$
|a(x, t)-a(x, s)| \leqslant N|t-s|
$$

for a.e. $x \in \Omega$ and every $s, t \in[0, T]$. For simplicity we define the family of operators

$$
\begin{aligned}
& A:[0, T] \rightarrow \mathcal{L}\left(H_{0}^{1}(\Omega), H^{-1}(\Omega)\right) \quad\langle A(t) u, v\rangle_{H^{-1}(\Omega) \times H_{0}^{1}(\Omega)}=\int_{\Omega}(a(x, t) \cdot D u(x), D v(x)) \mathrm{d} x \\
& \mathcal{A}: \mathcal{V} \rightarrow \mathcal{V}^{\prime} \quad \mathcal{A} u(t)=A(t) u(t) .
\end{aligned}
$$

Observe that under assumption (13) if we choose $a \in \mathcal{M}_{\Omega \times(0, T)}\left(\lambda_{0}, \Lambda_{0}, M, N\right)$ we can define $A^{\prime}:[0, T] \rightarrow$ $\mathcal{L}\left(H_{0}^{1}(\Omega), H^{-1}(\Omega)\right)$

$$
\left\langle A^{\prime}(t) u, v\right\rangle_{H^{-1} \times H_{0}^{1}}=\int_{\Omega}\left(a^{\prime}(x, t) \cdot D u(x), D v(x)\right) \mathrm{d} x \quad \text { where } a_{i j}^{\prime}(x, t)=\frac{\partial a_{i j}}{\partial t}(x, t)
$$

which by (13) turns out to be bounded. We recall now an existence result contained in [8] (Th. 3.8). Before we give the definition of solution.

Definition 2.5. Given $a \in \mathcal{M}_{\Omega \times(0, T)}\left(\lambda_{0}, \Lambda_{0}, M\right), r \in \mathcal{F}\left(C_{1}, C_{2}, \mu_{0}\right)$ with $\mu_{0}>-2 \lambda_{0}, f \in \mathcal{V}^{\prime}, \varphi \in L^{2}\left(\Omega_{+}^{r}(0)\right.$, $r(\cdot, 0)$ ) (see Rem. 2.4 for the definition of this space) a function $u \in \mathcal{W}$ is a solution of

$$
\begin{cases}\frac{\partial}{\partial t}(r u)-\operatorname{div}(a \cdot D u)=f & \text { on } \Omega \times(0, T) \\ u=0 & \text { in } \partial \Omega \times(0, T) \\ u(x, 0)=\varphi & \text { in } \Omega_{+}^{r}(0)\end{cases}
$$

if

$$
\begin{aligned}
& \left\langle(\mathcal{R} u)^{\prime}(t), v\right\rangle_{H^{-1}(\Omega) \times H_{0}^{1}(\Omega)}+\langle\mathcal{A} u(t), v\rangle_{H^{-1}(\Omega) \times H_{0}^{1}(\Omega)}=\langle f(t), v\rangle_{H^{-1}(\Omega) \times H_{0}^{1}(\Omega)} \\
& \int_{\Omega}(u(x, 0)-\varphi(x))^{2} r(x, 0) \mathrm{d} x=0
\end{aligned}
$$

for every $v \in H_{0}^{1}(\Omega)$ and for a.e. $t \in[0, T]$ and the initial datum makes sense in $L^{2}\left(\Omega_{+}^{r}(0), r(\cdot, 0)\right)$ thanks to Theorem 2.3. If $r \equiv 0$ the initial condition has no meaning and in this case a solution is a function $u \in \mathcal{V}$ such that

$$
\langle\mathcal{A} u(t), v\rangle_{H^{-1}(\Omega) \times H_{0}^{1}(\Omega)}=\langle f(t), v\rangle_{H^{-1}(\Omega) \times H_{0}^{1}(\Omega)}
$$

for every $v \in H_{0}^{1}(\Omega)$ and for a.e. $t \in[0, T]$.

Theorem 2.6. Consider $a \in \mathcal{M}_{\Omega \times(0, T)}\left(\lambda_{0}, \Lambda_{0}, M\right), r \in \mathcal{F}\left(C_{1}, C_{2}, \mu_{0}\right)$ with $\mu_{0}>-2 \lambda_{0}$. For every $f \in \mathcal{V}^{\prime}$ and $\varphi \in L^{2}\left(\Omega_{+}^{r}(0), r(\cdot, 0)\right)$ problem $(15)$ has a unique solution $u \in \mathcal{W}$ and there exists a constant $c=c\left(\mu_{0}, \lambda_{0}, \Lambda_{0}, C_{2}\right)$ 
(depending only on $\mu_{0}, \lambda_{0}, \Lambda_{0}, C_{2}$ ) such that

$$
\|u\|_{\mathcal{W}} \leqslant c\left[\|f\|_{\mathcal{V}^{\prime}}+\left\|\varphi(\cdot) r^{1 / 2}(\cdot, 0)\right\|_{L^{2}(\Omega)}\right]
$$

Statement of the problem - Fix $f \in \mathcal{V}^{\prime}$ and $\varphi \in L^{2}(\Omega)$ and consider

$$
\begin{aligned}
& \left(a_{h}\right)_{h} \subset \mathcal{M}_{\Omega \times(0, T)}\left(\lambda_{0}, \Lambda_{0}, M, N\right), \\
& \left(r_{h}\right)_{h} \subset \mathcal{F}\left(C_{1}, C_{2}, \mu_{0}\right) \\
& \mu_{0}>-2 \lambda_{0} .
\end{aligned}
$$

Assumption $\mu_{0}>-2 \lambda_{0}$ is required to have existence to problems (18) (see Th. 2.6).

Then we consider the sequence of elliptic-parabolic problems

$$
\begin{cases}\frac{\partial}{\partial t}\left(r_{h} u\right)-\operatorname{div}\left(a_{h} \cdot D u\right)=f & \text { on } \Omega \times(0, T) \\ u=0 & \text { in } \partial \Omega \times(0, T) \\ u(x, 0)=\varphi & \text { in } \Omega_{h,+}(0)\end{cases}
$$

where $\Omega_{h,+}(t):=\Omega_{+}^{r_{h}}(t)$. We consider $\varphi \in L^{2}(\Omega)$ so that problem (18) makes sense for every $h \in \mathbf{N}$, since $L^{2}(\Omega)$ is dense in $L^{2}\left(\Omega_{h,+}(0), r_{h}(\cdot, 0)\right)$ for every $h \in \mathbf{N}$ (see Rem. 2.4).

We want to study the asymptotic behaviour of the solutions $u_{h}$ when $h \rightarrow+\infty$ and characterise the limit problem.

The main difficulty is the lack of compactness of the solutions in $L^{2}\left(0, T ; L^{2}(\Omega)\right)$ which is natural in the classical case, i.e. when $r_{h} \equiv 1$.

In this framework the natural compactness result reads as follows in the theorem below (see Th. 2.14 and Th. 2.18 in [8]). Before we define

$$
\mathcal{W}_{h}=\left\{u \in \mathcal{V} \mid\left(r_{h} u\right)^{\prime} \in \mathcal{V}^{\prime}\right\}
$$

Theorem 2.7. Consider a sequence $\left(u_{h}\right)_{h}$ such that $u_{h} \in \mathcal{W}_{h}$ and $\left\|u_{h}\right\|_{\mathcal{W}_{h}} \leqslant c$ for a positive constant c. Then $\left(u_{h}\right)_{h}$ is relatively compact

(i) in $L^{2}\left(0, T ; L^{2}(\Omega)\right)$ if $r_{h} \rightarrow r$ in $L^{\infty}(\Omega \times(0, T))$-weakly $*$ and $r>0$ almost everywhere;

(ii) in $L^{2}\left(Q_{+} ; r\right)$, the completion of $C_{c}\left(Q_{+}\right)$with respect to the norm $\left\|u r^{1 / 2}\right\|_{L^{2}\left(Q_{+}\right)}$where $Q_{+}=\{(x, t) \in$ $\Omega \times(0, T) \mid r(x, t)>0\}$, if $r_{h} \rightarrow r$ in $L^{\infty}(\Omega \times(0, T))$ (strongly).

We by-pass the problem of the lack of compactness in $L^{2}\left(0, T ; L^{2}(\Omega)\right)$ via the following regularity result (see Th. 3.11 and Cor. 3.13 in [8]).

Theorem 2.8. Consider the problem (15). Assume that, besides to assumptions of Theorem 2.6, $\partial r / \partial t \in$ $\mathcal{F}\left(K_{1}, K_{2}\right)$, i.e. satisfies $(i)-(i v)$ of $(6)$ with constants $K_{1}, K_{2}$, and a satisfies $(13)$. Suppose moreover that $f \in$ $H^{1}\left(0, T ; H^{-1}(\Omega)\right)$ and that there exists $u_{0} \in H_{0}^{1}(\Omega)$ such that $u_{0}=\varphi$ in $\Omega_{+}^{r}(0)$ and $f(0)+\operatorname{div}\left(a(x, 0) D u_{0}(x)\right)-$ $\frac{\partial r}{\partial t}(x, 0)=r(x, 0) v(x)$ for some $v \in L^{2}\left(\Omega_{+}^{r}(0)\right)$. Then the solution u satisfies

$$
u \in H^{1}\left(0, T ; H_{0}^{1}(\Omega)\right) \quad \text { and } \quad\|u\|_{H^{1}\left(0, T ; H_{0}^{1}(\Omega)\right)} \leqslant c
$$

for a positive constant $c$ depending (only) on $\mu_{0}, \lambda_{0}, \Lambda_{0}, C_{2}, N, K_{2},\|f\|_{H^{1}\left(0, T ; H^{-1}(\Omega)\right)}$, $\left\|r^{1 / 2}(\cdot, 0) v\right\|_{L^{2}(\Omega)},\left\|r^{1 / 2}(\cdot, 0) u_{0}\right\|_{L^{2}(\Omega)}$. 


\section{Preliminary compactness Results}

In this section we will suppose more regularity on the sequence $\left(r_{h}\right)_{h}$ than we will require to state the main theorem (see Th. 4.5). Precisely in this section we require (see (12), (13) and (6))

$$
\begin{aligned}
& \left(a_{h}\right)_{h} \subset \mathcal{M}_{\Omega \times(0, T)}\left(\lambda_{0}, \Lambda_{0}, M, N\right), \\
& \left(r_{h}\right), \subset \mathcal{F}\left(C_{1}, C_{2}, \mu_{0}\right), \quad\left(\frac{\partial r_{h}}{\partial t}\right)_{h} \subset \mathcal{F}\left(K_{1}, K_{2}, \nu_{0}\right)
\end{aligned}
$$

for some constants $K_{1}, K_{2}, \nu_{0}$. Consider the problems

$$
\begin{cases}\frac{\partial}{\partial t}\left(r_{h} u\right)-\operatorname{div}\left(a_{h} \cdot D u\right)=g & \text { on } \Omega \times(0, T) \\ u=0 & \text { in } \partial \Omega \times(0, T) \\ u=\psi_{h} & \text { in } \Omega_{h,+}(0) \times\{0\}\end{cases}
$$

where $g \in H^{1}\left(0, T ; H^{-1}(\Omega)\right) \subset C\left([0, T] ; H^{-1}(\Omega)\right)$ and $\psi_{h}$ is the solution to

$$
\begin{cases}E_{h} w:=-\operatorname{div}\left(a_{h}(x, 0) \cdot D w(x)\right)+\frac{\partial r_{h}}{\partial t}(x, 0) w(x)=g(x, 0) & \text { in } \Omega \\ w=0 & \text { in } \partial \Omega\end{cases}
$$

where the linear, elliptic and bounded operator $E_{h}: H_{0}^{1}(\Omega) \rightarrow H^{-1}(\Omega)$ can be considered because $a_{h}$ and $r_{h}$ are continuous in the variable $t$.

Before stating the main result we recall the following lemma. Denote by $c_{P}$ the constant appearing in the Poincaré's inequality

$$
\int_{\Omega} u^{2}(x) \mathrm{d} x \leqslant c_{P} \int_{\Omega}|D u|^{2}(x) \mathrm{d} x, \quad u \in H_{0}^{1}(\Omega) .
$$

Lemma 3.1. Consider $a, a_{1}, a_{2} \ldots \in \mathcal{M}_{\Omega}\left(\lambda_{0}, \Lambda_{0}, M\right)$ and suppose $a_{h} \stackrel{E G}{\longrightarrow}$ a (see Def. 1.1). Consider a sequence of functions $\left(b_{h}\right)_{h} \subset L^{\infty}(\Omega), b_{h} \geqslant \gamma$ for every $h \in \mathbf{N}$ where $-\lambda_{0} / c_{P}<\gamma \leqslant 0$ and suppose $b_{h} \rightarrow b$ in $L^{\infty}(\Omega)$ weak*. Then for every $f \in H^{-1}(\Omega)$ it results that

$$
\begin{array}{ll}
w_{h} \rightarrow w & \text { in } L^{2}(\Omega) \\
a_{h} \cdot D w_{h} \rightarrow a \cdot D w & \text { in } L^{2}(\Omega)^{n}-\text { weak }
\end{array}
$$

where $w_{h}$ and $w$ denote respectively the solutions

$$
\left\{\begin{array} { l l } 
{ - \operatorname { d i v } ( a _ { h } \cdot D v ) + b _ { h } v = f } & { \text { in } \Omega } \\
{ v = 0 } & { \text { in } \partial \Omega }
\end{array} \quad \left\{\begin{array}{ll}
-\operatorname{div}(a \cdot D v)+b v=f & \text { in } \Omega \\
v=0 & \text { in } \partial \Omega .
\end{array}\right.\right.
$$

Proof. Since $-\lambda_{0} / c_{P}<\gamma \leqslant 0$ we have that

$$
\int_{\Omega} b_{h} v^{2} \mathrm{~d} x \geqslant \int_{\Omega} \gamma v^{2} \mathrm{~d} x \geqslant c_{P} \gamma \int_{\Omega}|D v|^{2} \mathrm{~d} x
$$

and then the elliptic operators $v \mapsto-\operatorname{div}\left(a_{h} \cdot D v\right)+b_{h} v$ are equicoercive. Since the solutions are compact in $L^{2}(\Omega)$ we have, up to a subsequence, that $-b_{h} w_{h}$ converges to $-b w$ weakly in $L^{2}(\Omega)$ and then strongly in $H^{-1}(\Omega)$. Then we obtain the thesis observing that $w_{h}$ solve the problems

$$
\begin{cases}-\operatorname{div}\left(a_{h} \cdot D v\right)=f_{h}:=f-b_{h} w_{h} & \text { in } \Omega \\ v=0 & \text { in } \partial \Omega\end{cases}
$$


Remark 3.2. As a consequence we have that, if $a_{h}(\cdot, 0) \stackrel{E G}{\longrightarrow} a(\cdot, 0)$ and $\left(r_{h}\right)_{h} \subset \mathcal{F}\left(C_{1}, C_{2}, \mu_{0}\right),\left(\frac{\partial r_{h}}{\partial t}\right)_{h} \subset$ $\mathcal{F}\left(K_{1}, K_{2}, \nu_{0}\right)$ for some $K_{1}, K_{2}, \nu_{0}$ and $\left|\frac{\partial r_{h}}{\partial t}\right|(x, 0) \leqslant \alpha$ for every $h \in \mathbf{N}$ with $0 \leqslant \alpha<\lambda_{0} / c_{P}$ are such that $r_{h} \rightarrow r$ in $L^{\infty}(\Omega \times(0, T)$ )-weak* (see also Rem. 2.1) equation (22) admits a unique solution since

$$
\int_{\Omega} u^{2}(x) \frac{\partial r_{h}}{\partial t}(x, 0) \mathrm{d} x \geqslant-\alpha \int_{\Omega} u^{2}(x) \mathrm{d} x>-\lambda_{0} \int_{\Omega}|D u|^{2}(x) \mathrm{d} x .
$$

The solutions $\psi_{h}$ of (22) satisfy

$$
\psi_{h} \rightarrow \psi \quad \text { in } L^{2}(\Omega), \quad a_{h} \cdot D \psi_{h} \rightarrow a \cdot D \psi \quad \text { in } L^{2}(\Omega)^{n} \text {-weak, }
$$

where $\psi$ is the solution of

$$
\begin{cases}E w:=-\operatorname{div}(a(x, 0) \cdot D w(x))+\frac{\partial r}{\partial t}(x, 0) w(x)=g(x, 0) & \text { in } \Omega \\ w=0 & \text { in } \partial \Omega .\end{cases}
$$

Now we state the first compactness result.

Lemma 3.3. Consider the problems (21) with the data $g \in H^{1}\left(0, T ; H^{-1}(\Omega)\right)$ and $\psi_{h}=E_{h}^{-1} g(0)$ and denote by $u_{h} \in \mathcal{W}_{h}$ the corresponding solutions. Then the sequence

$$
\left(u_{h}\right)_{h} \quad \text { is bounded in } H^{1}\left(0, T ; H_{0}^{1}(\Omega)\right) .
$$

As a consequence we obtain that

$$
\begin{aligned}
& \left(u_{h}\right)_{h} \quad \text { is relatively compact in } C\left([0, T] ; L^{2}(\Omega)\right) \text {, } \\
& \text { the sequence } t \mapsto \int_{\Omega} u_{h}^{2}(x, t) r_{h}(x, t) \mathrm{d} x \quad \text { is relatively compact in } C([0, T]) \text {. }
\end{aligned}
$$

Proof. Since $g(0)+\operatorname{div}\left(a_{h}(\cdot, 0) \cdot D \psi_{h}\right)-\frac{\partial r_{h}}{\partial t}(\cdot, 0) \psi_{h}=0$, hypotheses of Theorem 2.8 are satisfied. Then we have that the solutions satisfy the estimations

$$
\left\|u_{h}\right\|_{H^{1}\left(0, T ; H_{0}^{1}(\Omega)\right)} \leqslant c, \quad\left\|u_{h}\right\|_{C\left([0, T] ; H_{0}^{1}(\Omega)\right)} \leqslant c .
$$

We deduce that $\left(u_{h}\right)_{h}$ is equibounded in $C\left([0, T] ; H_{0}^{1}(\Omega)\right)$ and therefore the sets $\left\{u_{h}(t) \mid h \in \mathbf{N}\right\}$ are relatively compact in $L^{2}(\Omega)$ for every $t \in[0, T]$. Moreover

$$
u_{h}(t)-u_{h}(s)=\int_{s}^{t} u_{h}^{\prime}(\tau) \mathrm{d} \tau
$$

and then

$$
\left\|u_{h}(t)-u_{h}(s)\right\|_{H_{0}^{1}(\Omega)} \leqslant \int_{s}^{t}\left\|u_{h}^{\prime}(\tau)\right\|_{H_{0}^{1}(\Omega)} \mathrm{d} \tau \leqslant|t-s|^{1 / 2}\left\|u_{h}^{\prime}\right\|_{\mathcal{V}}
$$

so $\left(u_{h}\right)_{h}$ is also equicontinuous valued in $H_{0}^{1}(\Omega)$ (and in particular in $L^{2}(\Omega)$ ). Then by Lemma 1 in [12] we obtain $\left(u_{h}\right)_{h}$ relatively compact in $C\left([0, T] ; L^{2}(\Omega)\right)$.

To prove the second statement denote for simplicity by $\left(u_{h}\right)_{h}$ a subsequence converging in $C\left([0, T] ; L^{2}(\Omega)\right)$ and call $u$ the limit in $C\left([0, T] ; L^{2}(\Omega)\right)$. Consider the sequence $\left(r_{h}\right)_{h}$ : by Remark 2.1 we have the existence of 
$r \in \mathcal{F}\left(C_{1}, C_{2}, \mu_{0}\right)$ such that, up to a subsequence, $r_{h} \rightarrow r$ in $L^{\infty}(\Omega \times(0, T))$-weak*. Then, for $t, s \in[0, T]$,

$$
\begin{array}{r}
\left|\int_{\Omega} u_{h}^{2}(x, t) r_{h}(x, t) \mathrm{d} x-\int_{\Omega} u_{h}^{2}(x, s) r_{h}(x, s) \mathrm{d} x\right| \\
\quad \begin{array}{r}
\left|\int_{\Omega} u_{h}^{2}(x, t) r_{h}(x, t) \mathrm{d} x-\int_{\Omega} u_{h}^{2}(x, t) r_{h}(x, s) \mathrm{d} x\right| \\
\quad+\left|\int_{\Omega} u_{h}^{2}(x, t) r_{h}(x, s) \mathrm{d} x-\int_{\Omega} u_{h}^{2}(x, s) r_{h}(x, s) \mathrm{d} x\right| .
\end{array}
\end{array}
$$

By (6) and the continuity of $u_{h}:[0, T] \rightarrow L^{2}(\Omega)$ we infer that $t \mapsto \int_{\Omega} u_{h}^{2}(x, t) r_{h}(x, t) \mathrm{d} x$ is continuous. Moreover

$$
\begin{aligned}
& \left|\int_{\Omega} u_{h}^{2}(x, t) r_{h}(x, t) \mathrm{d} x-\int_{\Omega} u^{2}(x, t) r(x, t) \mathrm{d} x\right| \\
& \leqslant\left|\int_{\Omega} u_{h}^{2}(x, t) r_{h}(x, t) \mathrm{d} x-\int_{\Omega} u^{2}(x, t) r_{h}(x, t) \mathrm{d} x\right| \\
& +\left|\int_{\Omega} u^{2}(x, t) r_{h}(x, t) \mathrm{d} x-\int_{\Omega} u^{2}(x, t) r(x, t) \mathrm{d} x\right| \\
& \\
& \quad \leqslant\left\|r_{h}\right\|_{\infty} \int_{\Omega}\left|u_{h}^{2}(x, t)-u^{2}(x, t)\right| \mathrm{d} x+\left|\int_{\Omega} u^{2}(x, t)\left(r_{h}(x, t)-r(x, t)\right) \mathrm{d} x\right|
\end{aligned}
$$

by which we obtain the thesis.

Theorem 3.4. Consider a sequence of functions $v_{h} \in \mathcal{W}_{h}(h=1,2, \ldots)$, a function $v \in \mathcal{W}$, a constant $c_{1}$ such that

$$
\left\|v_{h}\right\|_{\mathcal{W}_{h}} \leqslant c_{1} \quad \text { for every } h, \quad v_{h} \rightarrow v \quad \text { in } C\left([0, T] ; L^{2}(\Omega)\right)
$$

and a sequence of vectorial functions $m_{h}, m \in L^{2}\left(0, T ;\left(L^{2}(\Omega)\right)^{n}\right)(h=1,2, \ldots)$, a constant $c_{2}$ such that

$$
\left\|m_{h}\right\|_{L^{2}\left(0, T ;\left(L^{2}(\Omega)\right)^{n}\right)} \leqslant c_{2} \quad m_{h} \rightarrow m \quad \text { in } L^{2}\left(0, T ;\left(L^{2}(\Omega)\right)^{n}\right) \text {-weak. }
$$

Moreover suppose that

Then

$$
\left(r_{h} v_{h}\right)^{\prime}-\operatorname{div}\left(m_{h}\right)=f \in \mathcal{V}^{\prime} \quad \text { in } C_{\mathrm{c}}^{1}(\Omega \times(0, T)) .
$$

$$
\left(m_{h}, D v_{h}\right) \rightarrow(m, D v) \quad \text { in } \mathcal{D}^{\prime}(\Omega \times(0, T)) .
$$

Proof. Fix a function $\varphi \in C_{\mathrm{c}}^{\infty}(\Omega \times(0, T))$ and multiply equation (26) by $v_{h} \varphi$. We obtain

$$
\int_{0}^{T} \int_{\Omega}\left(m_{h}, D v_{h}\right) \varphi \mathrm{d} x \mathrm{~d} t=\left\langle f-\left(r_{h} v_{h}\right)^{\prime}, v_{h} \varphi\right\rangle-\int_{0}^{T} \int_{\Omega}\left(m_{h}, D \varphi\right) v_{h} \mathrm{~d} x \mathrm{~d} t .
$$

Clearly $\left\langle f, v_{h} \varphi\right\rangle \rightarrow\langle f, v \varphi\rangle$ as $h \rightarrow \infty$ and $\int_{0}^{T} \int_{\Omega}\left(m_{h}, D \varphi\right) v_{h} \mathrm{~d} x \mathrm{~d} t \rightarrow \int_{0}^{T} \int_{\Omega}(m, D \varphi) v \mathrm{~d} x \mathrm{~d} t$. By (10) and since $\varphi$ has compact support in $\Omega \times(0, T)$ we have that

$$
-2\left\langle\left(r_{h} v_{h}\right)^{\prime}, v_{h} \varphi\right\rangle=\int_{0}^{T} \int_{\Omega} v_{h}^{2}(x, t) r_{h}(x, t) \frac{\partial \varphi}{\partial t}(x, t) \mathrm{d} x \mathrm{~d} t-\int_{0}^{T} \int_{\Omega} v_{h}^{2}(x, t) \varphi \frac{\partial r_{h}}{\partial t}(x, t) \mathrm{d} x \mathrm{~d} t
$$

which converges to

$$
\int_{0}^{T} \int_{\Omega} v^{2}(x, t) r(x, t) \frac{\partial \varphi}{\partial t}(x, t) \mathrm{d} x \mathrm{~d} t-\int_{0}^{T} \int_{\Omega} v^{2}(x, t) \varphi \frac{\partial r}{\partial t}(x, t) \mathrm{d} x \mathrm{~d} t=-2\left\langle(r v)^{\prime}, v \varphi\right\rangle .
$$


Then

$$
\int_{0}^{T} \int_{\Omega}\left(m_{h}, D v_{h}\right) \varphi \mathrm{d} x \mathrm{~d} t \rightarrow\left\langle f-(r v)^{\prime}, v \varphi\right\rangle-\int_{0}^{T} \int_{\Omega}(m, D \varphi) v \mathrm{~d} x \mathrm{~d} t
$$

and since, multiplying $(26)$ by $\phi \in C_{c}^{\infty}(\Omega \times(0, T))$ and taking the limit, we also have

$$
(r v)^{\prime}-\operatorname{div}(m)=f \quad \text { in } \mathcal{V}
$$

we obtain the thesis.

\section{The Definition of $G$-CONVERGence And the COMpaCtness Result}

In this section we give the main result, a compactness result with respect to $G$-convergence defined below (see Def. 4.3) for a sequence of operators (see (12) and (6))

$$
\begin{aligned}
& \mathcal{P}_{h} u=\frac{\partial}{\partial t}\left(r_{h} u\right)-\operatorname{div}\left(a_{h} \cdot D u\right), \\
& \left(a_{h}\right)_{h} \subset \mathcal{M}_{\Omega \times(0, T)}\left(\lambda_{0}, \Lambda_{0}, M, N\right), \quad\left(r_{h}\right)_{h} \subset \mathcal{F}\left(C_{1}, C_{2}, \mu_{0}\right) .
\end{aligned}
$$

The statement of this result is divided in two theorems. In the first one (Th. 4.1) we suppose the regularity required in the previous section, i.e. (20), and prove a partial result: the existence of a limit operator in divergence form. The second result (Th. 4.5) is a kind of uniqueness result: with less assumptions on the coefficients, i.e. satisfying the assumptions in (28), we will prove that the matrix defining the limit operator is independent of the sequence $\left(r_{h}\right)$.

Theorem 4.1. Consider a sequence $\left(a_{h}\right)_{h} \subset \mathcal{M}_{\Omega \times(0, T)}\left(\lambda_{0}, \Lambda_{0}, M, N\right)$ and $\left(r_{h}\right)_{h} \subset \mathcal{F}\left(C_{1}, C_{2}, \mu_{0}\right)$ with $\left(\frac{\partial r_{h}}{\partial t}\right)_{h} \subset$ $\mathcal{F}\left(K_{1}, K_{2}, \nu_{0}\right)$ and $\frac{\partial r_{h}}{\partial t}(x, 0)=0$ for every $h \in \mathbf{N}$. There exist a matrix $a \in \mathcal{M}_{\Omega \times(0, T)}\left(\lambda_{0}, M^{2} \Lambda_{0}, M \sqrt{\Lambda_{0} / \lambda_{0}}\right)$ and a function $r \in \mathcal{F}\left(C_{1}, C_{2}, \mu_{0}\right)$ such that for every $f \in \mathcal{V}^{\prime}$ and $\varphi \in L^{2}(\Omega)$ the solutions $u_{h}$ of problems (18), $h \in \mathbf{N}$, satisfy, up to a subsequence,

$$
u_{h} \rightarrow u \quad \text { in } L^{2}\left(0, T ; L^{2}(\Omega)\right) \quad \text { and } \quad a_{h} \cdot D u_{h} \rightarrow a \cdot D u \quad \text { in } L^{2}\left(0, T, L^{2}(\Omega)^{n}\right) \text {-weak }
$$

where $u$ is the solution of

$$
\left\{\begin{array}{lr}
\frac{\partial(r u)}{\partial t}-\operatorname{div}(a \cdot D u)=f & \text { in } \Omega \times(0, T) \\
u=0 & \text { in } \partial \Omega \times(0, T) \\
u=\varphi & \text { in } \Omega_{+}^{r}(0) \times\{0\} .
\end{array}\right.
$$

Remark 4.2. The result is true also if in (18) we consider a sequence of data $\left(f_{h}\right)_{h} \subset \mathcal{V}^{\prime},\left(f_{h}\right)_{h}$ relatively compact in $\mathcal{V}^{\prime}$, and $\left(\varphi_{h}\right)_{h} \subset L^{2}(\Omega), \varphi_{h}$ relatively compact in $L^{2}(\Omega)$.

Proof. First, by Remark 2.1, from $\left(r_{h}\right)_{h}$ we can extract a subsequence, still denoted by $\left(r_{h}\right)_{h}$, such that $r_{h} \rightarrow r$ in $L^{\infty}\left(\Omega \times(0, T)\right.$-weak $*$ and $r \in \mathcal{F}\left(C_{1}, C_{2}, \mu_{0}\right)$. Denote by $\mathcal{R}$ the operator defined by

$$
\mathcal{R}: L^{2}\left(0, T ; L^{2}(\Omega)\right) \rightarrow L^{2}\left(0, T ; L^{2}(\Omega)\right), \quad(\mathcal{R} u)(x, t)=r(x, t) u(x, t) .
$$

Analogously (to short) we denote by $\mathcal{R}_{h}$ the operators associated to $r_{h}$ and by $\mathcal{A}_{h}$ the operators associated to $a_{h}$ as defined in (14). Fix $X$ a countable and dense subset of $H^{1}\left(0, T ; H^{-1}(\Omega)\right)$ and $Y=\left\{y \in H_{0}^{1}(\Omega) \mid y=\right.$ $E^{-1} g(0)$ for $\left.g \in X\right\}$ where $E$ is the operator defined in Remark 3.2 (the $E G$-limit, up to a subsequence, of $\left\{a_{h}(\cdot, 0)\right\}_{h}$ ). Then consider $g \in X$ and $\psi=E^{-1} g(0)$ (and denote by $u_{h}(g, \psi)$ the solutions to the problems (21) with $\psi_{h}=E_{h}^{-1}(E \psi)$ where $E_{h}$ are the operators in (22). 
By Lemma 3.3 we have that the solutions $u_{h}(g, \psi)$ are compact in $C\left([0, T] ; L^{2}(\Omega)\right)$, and in particular $\psi_{h} \rightarrow \psi$ in $L^{2}(\Omega)$. Denote by $\mathcal{B}_{\mathcal{R}}(g, \psi)$ the limit in $C\left([0, T] ; L^{2}(\Omega)\right)$ of $u_{h}(g, \psi)$. In this way we have defined an operator in $Z=\left\{(g, \psi) \in X \times Y \mid \psi=E^{-1} g(0)\right\}$. $\mathcal{B}_{\mathcal{R}}$ is linear and continuous (see Th. 2.6). For every $h \in \mathbf{N}$ we have

$$
\left\|u_{h}(g, \psi)\right\|_{\mathcal{W}_{h}} \leqslant c\left[\|g\|_{\mathcal{V}^{\prime}}+\left\|\sqrt{r_{h}(\cdot, 0)} \psi_{h}\right\|_{L^{2}(\Omega)}\right] .
$$

Since $\left(r_{h} u_{h}\right)^{\prime} \rightarrow(r u)^{\prime}$ weakly in $\mathcal{V}^{\prime}$ and, by Lemma 3.3, $\sqrt{r_{h}(\cdot, 0)} \psi_{h} \rightarrow \sqrt{r(\cdot, 0)} \psi$ in $L^{2}(\Omega)$, by the lower semicontinuity of the norm we obtain

$$
\left\|\mathcal{B}_{\mathcal{R}}(g, \psi)\right\|_{\mathcal{W}} \leqslant c\left[\|g\|_{\mathcal{V}^{\prime}}+\|\sqrt{r(\cdot, 0)} \psi\|_{L^{2}(\Omega)}\right] .
$$

Since $Z$ is dense in $\mathcal{V}^{\prime} \times L^{2}\left(\Omega_{+}^{r}(0), r(\cdot, 0)\right)$, we can extend $\mathcal{B}_{\mathcal{R}}$ (and denote it in the same way)

$$
\mathcal{B}_{\mathcal{R}}: \mathcal{V}^{\prime} \times L^{2}\left(\Omega_{+}^{r}(0), r(\cdot, 0)\right) \rightarrow \mathcal{W} .
$$

Then we define

$$
\begin{aligned}
& \mathcal{K}_{\mathcal{R}}: \mathcal{V}^{\prime} \times L^{2}\left(\Omega_{+}^{r}(0), r(\cdot, 0)\right) \rightarrow \mathcal{V}^{\prime} \\
& \mathcal{K}_{\mathcal{R}} f=f-\frac{\mathrm{d}}{\mathrm{d} t}\left(\mathcal{R} \mathcal{B}_{\mathcal{R}}(f, \varphi)\right) \\
& \left\|\mathcal{K}_{\mathcal{R}}(f, \varphi)\right\|_{L^{2}\left(0, T ; H^{-1}(\Omega)\right)} \leqslant c^{\prime}\left[\|f\|_{\mathcal{V}^{\prime}}+\|\sqrt{r(\cdot, 0)} \varphi\|_{L^{2}(\Omega)}\right]
\end{aligned}
$$

So we have

$$
\frac{\mathrm{d}}{\mathrm{d} t}\left(\mathcal{R}_{h} u_{h}\right)+\mathcal{A}_{h} u_{h}=f \quad \text { and } \quad \frac{\mathrm{d}}{\mathrm{d} t}\left(\mathcal{R} \mathcal{B}_{\mathcal{R}}(f, \varphi)\right)+\mathcal{K}_{\mathcal{R}}(f, \varphi)=f .
$$

Multiplying the first in (32) by $u_{h}$ we have (where $\langle\cdot, \cdot\rangle$ denotes the duality between $\mathcal{V}^{\prime}$ and $\mathcal{V}$ )

$$
\begin{aligned}
\frac{1}{2} \int_{\Omega} u_{h}^{2}(x, T) r_{h}(x, T) \mathrm{d} x-\frac{1}{2} \int_{\Omega} \varphi^{2}(x) r_{h}( & x, 0) \mathrm{d} x+\int_{0}^{T} \int_{\Omega} \frac{\partial r_{h}}{\partial t} u_{h}^{2} \mathrm{~d} x \mathrm{~d} t \\
& +\int_{0}^{T} \int_{\Omega}\left(a_{h} \cdot D u_{h}, D u_{h}\right) \mathrm{d} x \mathrm{~d} t=\left\langle f, u_{h}\right\rangle
\end{aligned}
$$

and the second by $\mathcal{B}_{\mathcal{R}}(f, \varphi)$ we have

$$
\begin{array}{r}
\frac{1}{2} \int_{\Omega} \mathcal{B}_{\mathcal{R}}(f, \varphi)^{2}(x, T) r(x, T) \mathrm{d} x-\frac{1}{2} \int_{\Omega} \varphi^{2}(x) r(x, 0) \mathrm{d} x+\int_{0}^{T} \int_{\Omega} \frac{\partial r}{\partial t} \mathcal{B}_{\mathcal{R}}(f, \varphi)^{2} \mathrm{~d} x \mathrm{~d} t \\
+\left\langle\mathcal{K}_{\mathcal{R}}(f, \varphi), \mathcal{B}_{\mathcal{R}}(f, \varphi)\right\rangle=\left\langle f, \mathcal{B}_{\mathcal{R}}(f, \varphi)\right\rangle .
\end{array}
$$

Since

$$
\begin{aligned}
& \left\langle f, u_{h}\right\rangle \rightarrow\left\langle f, \mathcal{B}_{\mathcal{R}}(f, \varphi)\right\rangle \\
& \int_{\Omega} u_{h}^{2}(x, T) r_{h}(x, T) \mathrm{d} x \rightarrow \int_{\Omega} \mathcal{B}_{\mathcal{R}}(f, \varphi)^{2}(x, T) r(x, T) \mathrm{d} x \\
& \int_{\Omega} \varphi^{2}(x) r_{h}(x, 0) \mathrm{d} x \rightarrow \int_{\Omega} \varphi^{2}(x) r(x, 0) \mathrm{d} x \\
& \int_{0}^{T} \int_{\Omega} \frac{\partial r_{h}}{\partial t} u_{h}^{2} \mathrm{~d} x \mathrm{~d} t \rightarrow \int_{0}^{T} \int_{\Omega} \frac{\partial r}{\partial t} \mathcal{B}_{\mathcal{R}}(f, \varphi)^{2} \mathrm{~d} x \mathrm{~d} t
\end{aligned}
$$


we deduce that

$$
\int_{0}^{T} \int_{\Omega}\left(a_{h} \cdot D u_{h}, D u_{h}\right) \mathrm{d} x \mathrm{~d} t=\left\langle\mathcal{A}_{h} u_{h}, u_{h}\right\rangle \rightarrow\left\langle\mathcal{K}_{\mathcal{R}}(f, \varphi), \mathcal{B}_{\mathcal{R}}(f, \varphi)\right\rangle .
$$

The operator $\mathcal{B}_{\mathcal{R}}$ is injective: indeed if $\mathcal{B}_{\mathcal{R}}(f, \varphi)=0,\left\langle\mathcal{A}_{h} u_{h}, u_{h}\right\rangle \rightarrow 0$ and then $\left\|u_{h}\right\|_{\mathcal{V}} \rightarrow 0$. Since $\left(\mathcal{R}_{h} u_{h}\right)^{\prime} \rightarrow$ $\left(\mathcal{R B}_{\mathcal{R}}(f, \varphi)\right)^{\prime}$ weakly in $\mathcal{V}^{\prime}$ we have that $\mathcal{A}_{h} u_{h} \rightarrow \mathcal{K}_{\mathcal{R}}(f, \varphi)$ weakly in $\mathcal{V}^{\prime}$. Since $\mathcal{A}_{h}$ are equibounded and $u_{h} \rightarrow 0$ we conclude that $\mathcal{K}_{\mathcal{R}}(f, \varphi)=0$, i.e. $f-\frac{\mathrm{d}}{\mathrm{d} t}\left(\mathcal{R} \mathcal{B}_{\mathcal{R}}(f, \varphi)\right)=0$. Than $f=0$. Clearly, since $\mathcal{B}_{\mathcal{R}}(f, \varphi)=0$ and, by Theorem 2.3, we also have that $\varphi=0$ in $L^{2}\left(\Omega_{+}^{r}(0), r(\cdot, 0)\right)$.

Now we show that $\mathcal{B}_{\mathcal{R}}\left(\mathcal{V}^{\prime} \times L^{2}\left(\Omega_{+}^{r}(0), r(\cdot, 0)\right)\right)$ is dense in $\mathcal{V}$ : fix $g \in \mathcal{V}^{\prime}$ such that

$$
\left\langle g, \mathcal{B}_{\mathcal{R}}(f, \varphi)\right\rangle=0 \quad \text { for every } f \in \mathcal{V}, \varphi \in L^{2}\left(\Omega_{+}^{r}(0), r(\cdot, 0)\right) .
$$

In particular $\left\langle g, \mathcal{B}_{\mathcal{R}}(g, 0)\right\rangle=0$ and then

$$
\left\langle\frac{\mathrm{d}}{\mathrm{d} t}\left(\mathcal{R B}_{\mathcal{R}}(g, 0)\right)+\mathcal{K}_{\mathcal{R}}(g, 0), \mathcal{B}_{\mathcal{R}}(g, 0)\right\rangle=0
$$

i.e. $\left\langle\mathcal{K}_{\mathcal{R}}(g, 0), \mathcal{B}_{\mathcal{R}}(g, 0)\right\rangle \leqslant 0$. But by $(34)$ we know that $\left\langle\mathcal{K}_{\mathcal{R}}(g, 0), \mathcal{B}_{\mathcal{R}}(g, 0)\right\rangle \geqslant 0$, then it is zero. Therefore $u_{h}(g, 0) \rightarrow 0$ and as above we deduce that $g=0$.

Thus we can define the inverse of $\mathcal{B}_{\mathcal{R}}$ : if we define

$$
\begin{aligned}
& \mathcal{A}_{\mathcal{R}}: \mathcal{B}_{\mathcal{R}}\left(\mathcal{V}^{\prime} \times L^{2}\left(\Omega_{+}^{r}(0), r(\cdot, 0)\right)\right) \quad \longrightarrow \quad \mathcal{V}^{\prime} \\
& \mathcal{B}_{\mathcal{R}}(f, \varphi) \quad \mapsto \mathcal{K}_{\mathcal{R}}(f, \varphi),
\end{aligned}
$$

by density we can define an operator, still denoted by $\mathcal{A}_{\mathcal{R}}$,

$$
\mathcal{A}_{\mathcal{R}}: \mathcal{V} \rightarrow \mathcal{V}^{\prime}
$$

Now we need to define a last operator: for every $(g, \psi) \in(X, Y)$ the sequence $a_{h} \cdot D u_{h}(g, \psi)$ is bounded in $L^{2}\left(0, T ; L^{2}(\Omega)^{n}\right)$ and, up to a subsequence, weakly converges in $L^{2}\left(0, T ; L^{2}(\Omega)^{n}\right)$

$$
a_{h} \cdot D u_{h}(g, \psi) \rightarrow \mu(g, \psi)
$$

We have that

$$
\begin{aligned}
\left|a_{h}(x, t) \cdot D u_{h}(g, \psi)(x, t)\right| & =\sup _{|\eta|=1}\left(a_{h}(x, t) \cdot D u_{h}(x, t), \eta\right) \\
& \leqslant M \Lambda_{0}^{1 / 2}\left(a_{h}(x, t) \cdot D u_{h}(x, t), D u_{h}(x, t)\right)^{1 / 2} .
\end{aligned}
$$

By Lemma 7.8 in [2] and Theorem 3.4 we have that

$$
|\mu(g, \psi)| \leqslant M \Lambda_{0}^{1 / 2}\left(\mu(g, \psi), D \mathcal{B}_{\mathcal{R}}(g, \psi)\right)^{1 / 2}
$$

from which

Again by density, using (31), we can extend

$$
|\mu(g, \psi)| \leqslant M^{2} \Lambda_{0}\left|D \mathcal{B}_{\mathcal{R}}(g, \psi)\right| .
$$

$$
\mu: \mathcal{V}^{\prime} \times L^{2}\left(\Omega_{+}^{r}(0), r(\cdot, 0)\right) \rightarrow L^{2}\left(0, T ; L^{2}(\Omega)^{n}\right) .
$$

We define now $\mathcal{P}_{\mathcal{R}} u:=(\mathcal{R} u)^{\prime}+\mathcal{A}_{\mathcal{R}} u$ and

$$
\begin{array}{cccc}
M_{\mathcal{R}}: \mathcal{W} & \longrightarrow & L^{2}\left(0, T ; L^{2}(\Omega)^{n}\right) \\
u & \mapsto \mu\left(\mathcal{P}_{\mathcal{R}} u, P_{+}(0) u(0)\right)
\end{array}
$$


where $P_{+}(0) w$ is the restriction to $\Omega_{+}^{r}(0)$ of a function $w$ defined in $\Omega$. Observe that by (36), (32) and the definition of $\mathcal{A}_{\mathcal{R}}$ and $\mathcal{P}_{\mathcal{R}}$ one has, for every $u \in \mathcal{W}$,

$$
\left|M_{\mathcal{R}} u\right| \leqslant M^{2} \Lambda_{0}|D u|
$$

By definition we have that for every $v \in \mathcal{V}$

$$
\left\langle\mathcal{A}_{\mathcal{R}} u, v\right\rangle=\int_{0}^{T} \int_{\Omega}\left(M_{\mathcal{R}} u, D v\right) \mathrm{d} x \mathrm{~d} t .
$$

Now we want to construct a matrix $a$ such that for every $u, v \in \mathcal{V}$

$$
\int_{0}^{T} \int_{\Omega}\left(M_{\mathcal{R}} u, D v\right) \mathrm{d} x \mathrm{~d} t=\int_{0}^{T} \int_{\Omega}(a \cdot D u, D v) \mathrm{d} x \mathrm{~d} t .
$$

For this purpose fix $\omega \subset \subset \Omega$ and a function $\eta \in C^{1}(\bar{\Omega} \times[0, T])$ with $\eta(\cdot, t) \in C_{c}^{1}(\Omega)$ for every $t \in[0, T]$ such that $\eta=1$ on $\omega \times[0, T]$. Define $\phi_{\xi}(x, t)=(\xi, x) \eta(x, t)\left((\xi, x)\right.$ denotes the scalar product in $\left.\mathbf{R}^{n}\right)$. Finally, if $r$ is the function in $(30)$, define

$$
a_{r}(x, t) \cdot \xi=M_{\mathcal{R}}\left(\mathcal{P}_{\mathcal{R}} \phi_{\xi}, P_{+}(0) \phi_{\xi}(x, 0)\right) \quad \text { for }(x, t) \in \omega \times[0, T] .
$$

From (39) we obtain that

$$
\left\langle\mathcal{A}_{\mathcal{R}} u, v\right\rangle=\int_{0}^{T} \int_{\Omega}\left(a_{r} D u, D v\right) \mathrm{d} x \mathrm{~d} t
$$

and arguing as in [15], Theorem 3, we obtain

$$
\lambda_{0}|\xi|^{2} \leqslant\left(a_{r}(x, t) \cdot \xi, \xi\right), \quad\left(a_{r}(x, t) \cdot \xi, \eta\right) \leqslant M \Lambda_{0}^{1 / 2}\left(a_{r}(x, t) \cdot \xi, \xi\right)^{1 / 2}|\eta|^{1 / 2}
$$

for a.e. $(x, t) \in \Omega \times(0, T)$ and for every $\xi, \eta \in \mathbf{R}^{n}$. Then also for the operator $\mathcal{A}_{\mathcal{R}}$ we have

$$
\lambda_{0}\|u\|_{\mathcal{V}} \leqslant\left\langle\mathcal{A}_{\mathcal{R}} u, u\right\rangle \text { and }\left|\left\langle\mathcal{A}_{\mathcal{R}} u, v\right\rangle\right| \leqslant M^{2} \Lambda_{0}\|u\|_{\mathcal{V}}\|v\|_{\mathcal{V}} .
$$

Definition 4.3. Consider a sequence $\left(a_{h}\right)_{h} \subset \mathcal{M}_{\Omega \times(0, T)}\left(\lambda_{0}, \Lambda_{0}, M, N\right)$. We say that

$$
a_{h} \stackrel{G}{\longrightarrow} a \quad \text { in } \Omega \times(0, T)
$$

if for every $f \in \mathcal{V}^{\prime}$, for every $\varphi \in L^{2}(\Omega)$ and for every $\left(r_{h}\right)_{h} \subset \mathcal{F}\left(C_{1}, C_{2}, \mu_{0}\right)$ and $r \in \mathcal{F}\left(C_{1}, C_{2}, \mu_{0}\right)$ with $\mu_{0}>-2 \lambda_{0}$ and

$$
r_{h} \rightarrow r \quad L^{\infty}(\Omega \times(0, T)) \text {-weak* }
$$

it results that

$$
\begin{array}{ll}
u_{h} \rightarrow u & \text { in } L^{2}\left(0, T, L^{2}(\Omega)\right) \\
a_{h} \cdot D u_{h} \rightarrow a \cdot D u & \text { in } L^{2}\left(0, T, L^{2}(\Omega)^{n}\right) \text {-weak, }
\end{array}
$$

where $u_{h}$ and $u$ denote respectively the solutions of

$$
\left\{\begin{array} { l r } 
{ \frac { \partial } { \partial t } ( r _ { h } v ) - \operatorname { d i v } ( a _ { h } \cdot D v ) = f \text { in } \Omega \times ( 0 , T ) } \\
{ v = 0 } & { \text { in } \partial \Omega \times ( 0 , T ) } \\
{ v = \varphi } & { \text { in } \Omega _ { h , + } ( 0 ) \times \{ 0 \} }
\end{array} \quad \left\{\begin{array}{lr}
\frac{\partial}{\partial t}(r v)-\operatorname{div}(a \cdot D v)=f \text { in } \Omega \times(0, T) \\
v=0 \\
v=\varphi & \text { in } \partial \Omega \times(0, T)
\end{array}\right.\right.
$$


Before stating the main result we need a short and preliminary lemma regarding the following problems

$$
\begin{cases}A_{h} u=-\operatorname{div}\left(a_{h} \cdot D u\right)=f & \text { in } \Omega \times(0, T) \\ u=0 & \text { in } \partial \Omega \times(0, T)\end{cases}
$$

where $\left(a_{h}\right)_{h} \subset \mathcal{M}_{\Omega \times(0, T)}\left(\lambda_{0}, \Lambda_{0}, M, N\right)$.

Lemma 4.4. Consider the problems (41) with $f \in H^{1}\left(0, T ; H^{-1}(\Omega)\right)$ and suppose the solutions $u_{h}$ satisfy

$$
u_{h} \rightarrow u \quad \text { in } L^{2}\left(0, T ; L^{2}(\Omega)\right) \quad \text { and } \quad a_{h} \cdot D u_{h} \rightarrow a \cdot D u \quad \text { in } L^{2}\left(0, T, L^{2}(\Omega)^{n}\right) \text {-weak }
$$

where $u$ is the solution of

$$
\begin{cases}A u=-\operatorname{div}(a \cdot D u)=f & \text { in } \Omega \times(0, T) \\ u=0 & \text { in } \partial \Omega \times(0, T) .\end{cases}
$$

Then $t \mapsto a(\cdot, t)$ is continuous $\left(\lim _{t \rightarrow s}\|\mid a(\cdot, t)-a(\cdot, s)\|_{L^{\infty}(\Omega)}=0\right.$ for every $\left.s \in[0, T]\right)$ and $a_{h}(\cdot, t) \stackrel{E G}{\longrightarrow} a(\cdot, t)$ for every $t \in[0, T]$.

Proof. Let $u_{h}$ be the solution of (41), where $A_{h}: \mathcal{V} \rightarrow \mathcal{V}^{\prime}$. Observe that $u_{h}(t)$ solves $A_{h}(t) u_{h}(t)=f(t)$ for almost every $t \in[0, T]$. Then, by Theorem $2.8,\left(u_{h}\right)_{h}$ is equibounded and equicontinuous in $[0, T]$ with respect to the $H_{0}^{1}(\Omega)$-norm and moreover $u_{h} \rightarrow u$ in $C\left([0, T] ; L^{2}(\Omega)\right)$. Then we have for every $t \in[0, T]$

$$
A_{h}^{-1}(t) f(t) \rightarrow A^{-1}(t) f(t) \quad \text { in } L^{2}(\Omega) .
$$

Moreover we have that, for every $\Phi \in L^{2}(\Omega)^{n}$, the functions $t \mapsto \int_{\Omega}\left(a_{h}(x, t) D u_{h}(x, t), \Phi\right) \mathrm{d} x$ are equicontinuous and equibounded. Indeed

$$
\begin{aligned}
& \left|\int_{\Omega}\left(a_{h}(x, t) D u_{h}(x, t), \Phi\right) \mathrm{d} x-\int_{\Omega}\left(a_{h}(x, s) D u_{h}(x, s), \Phi\right) \mathrm{d} x\right| \leqslant \\
& \quad \leqslant N|t-s| \int_{\Omega}\left|\left(D u_{h}(x, t), \Phi\right)\right| \mathrm{d} x+M \Lambda_{0}\left[\int_{\Omega}\left|D u_{h}(x, t)-D u_{h}(x, s)\right|^{2} \mathrm{~d} x \int_{\Omega}|\Phi|^{2} \mathrm{~d} x\right]^{1 / 2} .
\end{aligned}
$$

By equicontinuity of $\left(u_{h}\right)_{h}$ in $H_{0}^{1}(\Omega)$ we conclude that the sequence $t \mapsto \int_{\Omega}\left(a_{h}(x, t) D u_{h}(x, t), \Phi\right) \mathrm{d} x$ is relatively compact in $C\left([0, T]\right.$ ), and consequently there is a subsequence $\left(a_{h_{j}}\right)_{j}$ (since $H_{0}^{1}(\Omega)$ is separable) and a vectorial function $V$ such that for every $\Phi \in L^{2}(\Omega)^{n}$ and $\eta \in C[0, T]$, one has

$$
\int_{0}^{T} \eta(t)\left[\int_{\Omega}\left(a_{h_{j}}(x, t) D u_{h_{j}}(x, t), \Phi(x)\right) \mathrm{d} x\right] \mathrm{d} t \rightarrow \int_{0}^{T} \eta(t)\left[\int_{\Omega}(V(x, t), \Phi(x)) \mathrm{d} x\right] \mathrm{d} t .
$$

By assumptions we conclude that $V=a \cdot D u$. Finally, since this can be derived for every subsequence of $\left(a_{h} \cdot D u_{h}\right)_{h}$ we conclude that the whole sequence $a_{h}(\cdot, t) \stackrel{E G}{\longrightarrow} a(\cdot, t)$ for every $t \in[0, T]$. The continuity follows by Theorem 2.4 in [3].

Theorem 4.5. The class $\mathcal{M}_{\Omega \times(0, T)}\left(\lambda_{0}, \Lambda_{0}, M, N\right)$ of matrices satisfying (2) is relatively compact with respect to G-convergence.

Proof. Consider $\left(a_{h}\right)_{h} \subset \mathcal{M}_{\Omega \times(0, T)}\left(\lambda_{0}, \Lambda_{0}, M, N\right), f \in \mathcal{V}^{\prime}$ and problems (18) with $r_{h} \equiv 0$ for every $h \in \mathbf{N}$ (see Def. (2.5)) and denote by $w_{h}$ the corresponding solutions. By Theorem 4.1 we have that there exists a subsequence $\left(a_{h_{k}}\right)_{k}$ and a matrix

$$
\bar{a} \in \mathcal{M}_{\Omega \times(0, T)}\left(\lambda_{0}, M^{2} \Lambda_{0}, M \sqrt{\Lambda_{0} / \lambda_{0}}\right)
$$


such that the sequence $\left(w_{h_{k}}\right)_{k}$ satisfies

$$
w_{h_{k}} \rightarrow w \quad \text { in } L^{2}\left(0, T ; L^{2}(\Omega)\right) \quad \text { and } \quad a_{h_{k}} \cdot D w_{h_{k}} \rightarrow \bar{a} \cdot D w \quad \text { in } L^{2}\left(0, T ; L^{2}(\Omega)^{n}\right) \text {-weak }
$$

where $w$ is the solution of

$$
\begin{cases}-\operatorname{div}(\bar{a}(x, t) \cdot D u(x, t))=f(x, t) & \text { in } \Omega \times(0, T) \\ u=0 & \text { in } \partial \Omega \times(0, T) .\end{cases}
$$

Now consider a sequence $\left(r_{h}\right)_{h} \subset \mathcal{F}\left(C_{1}, C_{2}, \mu_{0}\right)$ with $\left(\frac{\partial r_{h}}{\partial t}\right)_{h} \subset \mathcal{F}\left(K_{1}, K_{2}, \nu_{0}\right)$. Up to a subsequence

$$
r_{h} \rightarrow r \quad \text { and } \quad \frac{\partial r_{h}}{\partial t} \rightarrow \frac{\partial r}{\partial t} \quad \text { in } L^{\infty}(\Omega \times(0, T)) \text {-weak* }
$$

for a $r \in \mathcal{F}\left(C_{1}, C_{2}, \mu_{0}\right)$ with $\frac{\partial r}{\partial t} \in \mathcal{F}\left(K_{1}, K_{2}, \nu_{0}\right)$ (see Rem. 2.1). Consider this function $r$ and the matrix $\bar{a}$ in $(42)$ and define the operators in $\mathcal{L}\left(H_{0}^{1}(\Omega), H^{-1}(\Omega)\right)$

$$
E_{h}(t) u:=-\operatorname{div}\left(a_{h}(\cdot, t) \cdot D u\right)+\frac{\partial r_{h}}{\partial t}(\cdot, t) u, \quad E(t) u:=-\operatorname{div}(\bar{a}(\cdot, t) \cdot D u)+\frac{\partial r}{\partial t}(\cdot, t) u
$$

for every $t \in[0, T]$ (this is possible thanks to Lem. 4.4). Now consider $f \in \mathcal{V}^{\prime}$ and $\varphi \in L^{2}(\Omega)$, the following problems

$$
\begin{cases}\frac{\partial}{\partial t}\left(r_{h} u\right)-\operatorname{div}\left(a_{h} \cdot D u\right)=f & \text { on } \Omega \times(0, T) \\ u=0 & \text { in } \partial \Omega \times(0, T) \\ u=\varphi & \text { in } \Omega_{h,+}(0) \times\{0\}\end{cases}
$$

and let $u_{h}$ be the corresponding solutions. For every $\epsilon>0$ we can find $\psi \in H_{0}^{1}(\Omega)$ and $g \in H^{1}\left(0, T ; H^{-1}(\Omega)\right)$ such that

$$
E(0) \psi=g(0), \quad\|\varphi-\psi\|_{L^{2}(\Omega)}<\epsilon / 2, \quad\|f-g\|_{\mathcal{V}^{\prime}}<\epsilon / 2
$$

and define $\psi_{h}:=E_{h}(0)^{-1} g(0)$. We have that the sequence of the solutions $\left(v_{h}\right)_{h}$ of

$$
\begin{cases}\frac{\partial}{\partial t}\left(r_{h} u\right)-\operatorname{div}\left(a_{h} \cdot D u\right)=g & \text { on } \Omega \times(0, T) \\ u=0 & \text { in } \partial \Omega \times(0, T) \\ u=\psi_{h} & \text { in } \Omega_{h,+}(0) \times\{0\}\end{cases}
$$

satisfies, by Theorem 2.6,

$$
\begin{aligned}
& \left\|u_{h}-v_{h}\right\|_{L^{2}\left(0, T ; L^{2}(\Omega)\right)} \leqslant c\left(C_{1}\right) \epsilon \\
& \left\|a_{h} \cdot D u_{h}-a_{h} \cdot D v_{h}\right\|_{L^{2}\left(0, T ; L^{2}(\Omega)^{n}\right)} \leqslant c\left(M, \Lambda_{0}\right) \epsilon
\end{aligned}
$$

and moreover is relatively compact in $C\left([0, T] ; L^{2}(\Omega)\right)$ by Lemma 3.3. Denote by $\mathcal{R}_{h}$ the operator in $\mathcal{L}\left(L^{2}\left(0, T ; L^{2}(\Omega)\right)\right)$ defined by $\mathcal{R}_{h} u=r_{h} u$ and $\mathcal{R}$ the operator in $\mathcal{L}\left(L^{2}\left(0, T ; L^{2}(\Omega)\right)\right)$ defined by $\mathcal{R} u=r u$. By Theorem 2.8 we have that, in particular, $\left(\mathcal{R}_{h} v_{h}\right)^{\prime}$ is relatively compact in $C\left([0, T] ; H^{-1}(\Omega)\right)$. Then, up to a subsequence,

$$
\left(\mathcal{R}_{h} v_{h}\right)^{\prime} \rightarrow(\mathcal{R} v)^{\prime} \quad \text { in } C\left([0, T] ; H^{-1}(\Omega)\right)
$$


Observe then that $v_{h}$ solves the following problem in $\mathcal{V}$

$$
\begin{cases}-\operatorname{div}\left(a_{h}(x, t) \cdot D v(x, t)\right)=g(x, t)-\frac{\partial}{\partial t}\left(r_{h}(x, t) v_{h}(x, t)\right)=: g_{h}(x, t) & \text { in } \Omega \times(0, T) \\ v=0 & \text { in } \partial \Omega \times(0, T)\end{cases}
$$

with, up to a subsequence, $g_{h} \rightarrow g-\frac{\partial(r v)}{\partial t}$ strongly in $\mathcal{V}^{\prime}$ and moreover $v_{h}(x, 0)=\psi_{h}(x)$ in $\Omega_{h,+}(0)$. By Remark 4.2 we have that if we consider the subsequence $\left(a_{h_{k}}\right)_{k}$ (or if necessary extracting from this another subsequence because of $\left.\left(r_{h}\right)_{h}\right)$ we have that

$$
\begin{aligned}
& v_{h_{k}} \rightarrow v \quad \text { in } C\left([0, T] ; L^{2}(\Omega)\right) \quad \text { and } \\
& a_{h_{k}} \cdot D v_{h_{k}} \rightarrow \bar{a} \cdot D v \quad \text { in } L^{2}\left(0, T ; L^{2}(\Omega)^{n}\right) \text {-weak }
\end{aligned}
$$

where $v$ is the solution of

$$
\begin{cases}-\operatorname{div}(\bar{a}(x, t) \cdot D v(x))=f(x, t)-\frac{\partial}{\partial t}(r(x, t) v(x, t)) & \text { in } \Omega \times(0, T) \\ v=0 & \text { in } \partial \Omega \times(0, T)\end{cases}
$$

and moreover, by Lemma 3.3,

$$
\left(r_{h_{k}}(x, 0)\right)^{1 / 2} v_{h_{k}}(x, 0) \rightarrow(r(x, 0))^{1 / 2} v(x, 0) \quad \text { in } L^{2}(\Omega) .
$$

Since $\left(r_{h_{k}}(x, 0)\right)^{1 / 2} v_{h_{k}}(x, 0)=\left(r_{h_{k}}(x, 0)\right)^{1 / 2} \psi_{h_{k}}(x)$ and $\psi_{h_{k}} \rightarrow \psi$ in $L^{2}(\Omega)$ (by Lemma 3.1 and Remark 3.2) we conclude that $v$ solves

$$
\begin{cases}\frac{\partial}{\partial t}(r v)-\operatorname{div}(\bar{a} \cdot D v)=g & \text { on } \Omega \times(0, T) \\ v=0 & \text { in } \partial \Omega \times(0, T) \\ v=\psi & \text { in } \Omega_{+}^{r}(0) \times\{0\} .\end{cases}
$$

Now consider the solution $u$ to the problem

$$
\begin{cases}\frac{\partial}{\partial t}(r u)-\operatorname{div}(\bar{a} \cdot D u)=f & \text { on } \Omega \times(0, T) \\ u=0 & \text { in } \partial \Omega \times(0, T) \\ u=\varphi & \text { in } \Omega_{+}^{r}(0) \times\{0\} .\end{cases}
$$

Then taking the limit in

$$
\left\|u_{h_{k}}-u\right\|_{\mathcal{V}} \leqslant\left\|u_{h_{k}}-v_{h_{k}}\right\| \mathcal{V}+\left\|v_{h_{k}}-v\right\|_{\mathcal{V}}+\|v-u\|_{\mathcal{V}}
$$

we obtain thanks to (43) and (44) that $\lim _{k}\left\|u_{h_{k}}-u\right\|_{\mathcal{V}} \leqslant c \epsilon$. In the same way we obtain that

$$
\left|\lim _{k} \int_{0}^{T} \int_{\Omega}\left[\left(a_{h_{k}} \cdot D u_{h_{k}}, \Phi\right)-(\bar{a} \cdot D u, \Phi)\right] \mathrm{d} x \mathrm{~d} t\right| \leqslant c \epsilon
$$

for every $\Phi \in C_{c}^{1}(\Omega)^{n}$. This concludes the proof that the matrix $\bar{a}$ does not depend on the sequence $\left(r_{h}\right)_{h}$ and on the limit $r$.

Now we show that the hypothesis $\left(\frac{\partial r_{h}}{\partial t}\right)_{h} \subset \mathcal{F}\left(K_{1}, K_{2}, \nu_{0}\right)$ can be dropped. Then suppose only $\left(r_{h}\right)_{h} \subset$ $\mathcal{F}\left(C_{1}, C_{2}, \mu_{0}\right)$, let $r$ be the limit of $r_{h}$ as before and denote by $u_{h}$ the solutions to problems (18). By Lemma 2.2 for 
every $h \in \mathbf{N}$ we can consider a sequence of functions $v_{h} \in \mathcal{V}$ and a sequence of functions $s_{h},\left(s_{h}\right)_{h} \subset \mathcal{F}\left(C_{1}, C_{2}, \mu_{0}\right)$ and $\left(\frac{\partial s_{h}}{\partial t}\right)_{h} \subset \mathcal{F}\left(C_{2}, K,-K\right)$, with $K$ independent of $h$, such that (we denote by $\mathcal{S}_{h}$ the operator defined by $\mathcal{S}_{h} w=s_{h} w$ and recall the definition of $\mathcal{W}_{h}$ is given in (19))

$$
\left\|u_{h}-v_{h}\right\|_{\mathcal{W}_{h}}<\frac{1}{h}, \quad\left\|\left(\mathcal{R}_{h} u_{h}\right)^{\prime}-\left(\mathcal{S}_{h} v_{h}\right)^{\prime}\right\|_{\mathcal{V}^{\prime}}<\frac{1}{h}, \quad \text { and moreover } S_{h}^{\prime}(0)=0 .
$$

By the first of these estimates and by (11) we obtain that

$$
\int_{\Omega}\left|u_{h}(x, 0)-v_{h}(x, 0)\right|^{2} r_{h}(x, 0) \mathrm{d} x<c \frac{1}{h^{2}}
$$

and since $u_{h}(x, 0)=\varphi(x)$ in $\Omega_{h,+}$ we have

$$
\int_{\Omega}\left|\varphi(x)-v_{h}(x, 0)\right|^{2} r_{h}(x, 0) \mathrm{d} x<c \frac{1}{h^{2}} .
$$

Observe that the function $v_{h}$ solve the following problem

$$
\left\{\begin{array}{l}
\left(\mathcal{S}_{h} w\right)^{\prime}+\mathcal{A}_{h} w=f_{h}:=f+\left(\mathcal{S}_{h} v_{h}\right)^{\prime}-\left(\mathcal{R}_{h} u_{h}\right)^{\prime}+\mathcal{A}_{h} v_{h}-\mathcal{A}_{h} u_{h} \\
P_{+, h}(0) w(0)=\varphi_{h}:=\varphi+\left(v_{h}(0)-u_{h}(0)\right) .
\end{array}\right.
$$

By Lemma 2.2 we have that $f_{h} \rightarrow f$ in $\mathcal{V}^{\prime}$ and finally, by Remark 4.2 since $\mathcal{S}_{h}$ satisfy hypotheses of Theorem 4.1, we obtain, taking the limit, that there exist a function $v \in \mathcal{V}$ and a function $s \in \mathcal{F}\left(C_{1}, C_{2}, \mu_{0}\right)$ such that, up to a subsequence,

$$
\begin{aligned}
& v_{h} \rightarrow v \quad \text { in } L^{2}\left(0, T ; L^{2}(\Omega)\right) \\
& a_{h} \cdot D v_{h} \rightarrow \bar{a} \cdot D v \quad \text { in } L^{2}\left(0, T ; L^{2}(\Omega)^{n}\right) \text {-weak, }
\end{aligned}
$$

where $v$ solves the problem

$$
\begin{cases}\frac{\partial}{\partial t}(s v)-\operatorname{div}(\bar{a} \cdot D v)=f & \text { on } \Omega \times(0, T) \\ v=0 & \text { in } \partial \Omega \times(0, T) \\ v=\varphi & \text { in } \Omega_{+}^{s}(0) \times\{0\} .\end{cases}
$$

By estimations above we deduce that $v=u$ and that $s=r$ and that

$$
\begin{aligned}
& u_{h} \rightarrow u \quad \text { in } L^{2}\left(0, T ; L^{2}(\Omega)\right) \\
& a_{h} \cdot D u_{h} \rightarrow \bar{a} \cdot D u \quad \text { in } L^{2}\left(0, T ; L^{2}(\Omega)^{n}\right) \text {-weak }
\end{aligned}
$$

and then the result is completely proved.

\section{EXAmples}

In this section we present first some possible choices in the class $\mathcal{F}\left(C_{1}, C_{2}, \mu_{0}\right)$, then some particular cases of $G$-convergence: in Subsection 5.2 the classical variational convergences, in 5.3 the result in homogenization, in 5.4 the singular perturbations, in which the convergence of the solutions is stronger (Lem. 5.1). 


\subsection{Examples of admissible $r$}

1) $r \equiv r(x)$ - Besides $r \equiv 1$ and $r \equiv 0$, one can consider more general situations. Every non-negative $r \in L^{\infty}(\Omega)$, i.e. $r=r(x)$ depending only on $x$, belongs to the class $\mathcal{F}\left(C_{1}, C_{2}, \mu_{0}\right)$ with $C_{1}=\|r\|_{\infty}$ and $C_{2}=\mu_{0}=0$. Then also

$$
r(x)= \begin{cases}1 & \text { in a Cantor type set } \Omega_{+} \\ 0 & \text { outside } \Omega_{+},\end{cases}
$$

$\Omega_{+}$with positive measure, is admitted.

2) $r=r(t)$ - Every regular function satisfying suitable bounds can be admitted. Since

$$
t \mapsto \int_{\Omega} u(x) v(x) r(t) \mathrm{d} x
$$

is required to be absolutely continuous we will require $r \in W^{1, \infty}(0, T)$. In this way

$$
\frac{\mathrm{d}}{\mathrm{d} t} \int_{\Omega} u(x) v(x) r(t) \mathrm{d} x=r^{\prime}(t) \int_{\Omega} u(x) v(x) \mathrm{d} x
$$

and it is sufficient to require (remember $\mu_{0} \leqslant 0$ )

$$
0 \leqslant r \leqslant C_{1}, \quad \frac{\mu_{0}}{c_{P}} \leqslant r^{\prime}(t) \leqslant C_{2},
$$

where $c_{P}$ is the constant appearing in (23), to have $r \in \mathcal{F}\left(C_{1}, C_{2}, \mu_{0}\right)$.

As particular case we want to observe that if $r(0)=0$ and $r(t)>0$ for every $t>0$ the initial condition is not needed. For example, if $r(t)=t$ the problem

$$
\left\{\begin{array}{lr}
\frac{\partial}{\partial t}(t v)-\operatorname{div}(a(x, t) \cdot D v)=f & \text { in } \Omega \times(0, T) \\
v=0 & \text { in } \partial \Omega \times(0, T)
\end{array}\right.
$$

has a unique solution (without any condition at time $t=0$ ).

3) $r=r(x, t)$ - If $r, \frac{\partial r}{\partial t} \in L^{\infty}(\Omega \times(0, T))$ then $r \in \mathcal{F}\left(C_{1}, C_{2}, \mu_{0}\right)$ with $C_{1}=\|r\|_{\infty}$ and $C_{2}=\left\|\frac{\partial r}{\partial t}\right\|_{\infty}$.

Precisely, if $c_{P}$ is the constant appearing in (23), $r$ can be choosen such that

$$
0 \leqslant r \leqslant C_{1}, \quad \frac{\mu_{0}}{c_{P}} \leqslant \frac{\partial r}{\partial t} \leqslant C_{2}
$$

$\left(C_{2} \geqslant 0, \mu_{0} \leqslant 0\right)$.

Also functions $r$ for which $\frac{\partial r}{\partial t} \notin L^{\infty}(\Omega \times(0, T))$ can be considered. For example if

$$
r(x, t)=\chi_{A}(x, t), \quad A \subset \Omega \times(0, T) .
$$

In this case, if we denote $\Omega_{+}(t)=\{x \in \Omega \mid r(\cdot, t)>0\}$, we need

$$
t \mapsto \int_{\Omega_{+}(t)} u(x) v(x) \mathrm{d} x \quad \text { differentiable. }
$$

We refer to [8] for more details and to [6] (Prop. 3, Sect. 3.4.4) for differentiability of (45). 


\subsection{Variational convergences}

4) If $r_{h} \equiv 0$ for every $h$ we have a result for a class of elliptic operators and we have that

$$
a_{h} \stackrel{G}{\longrightarrow} a \text { in } \Omega \times(0, T) \quad \Longleftrightarrow \quad a_{h}(\cdot, t) \stackrel{E G}{\longrightarrow} a(\cdot, t) \quad \text { in } \Omega \text { for a.e. } t \in[0, T] .
$$

5) If $r_{h} \equiv 1$ for every $h$ we have a result for a class of parabolic operators and we have that

$$
a_{h} \stackrel{G}{\longrightarrow} a \quad \text { in } \Omega \times(0, T) \quad \Longleftrightarrow \quad a_{h} \stackrel{P G}{\longrightarrow} a \text { in } \Omega \times(0, T) .
$$

6) Suppose $a_{h}$ to be symmetric matrices. Then, choosing $r_{h} \equiv 0$ and using the classical result (see for instance [4] for the definition of $\Gamma$-convergence) we obtain that

$$
a_{h} \stackrel{G}{\longrightarrow} a \quad \text { in } \Omega \times(0, T) \quad \Longleftrightarrow \quad a_{h}(\cdot, t) \stackrel{\Gamma}{\longrightarrow} a(\cdot, t) \quad \text { in } \Omega \text { for a.e. } t \in[0, T] .
$$

\subsection{Homogenization}

7) If $r(x)$ is a $Q$-periodic function in the variable $x$ and $a_{i j}$, the entries of a matrix $a \in \mathcal{M}_{\Omega \times(0, T)}\left(\lambda_{0}, \Lambda_{0}, M\right)$, are $Q$-periodic in the variable $x, Q$ cube of $\mathbf{R}^{n}$, then the solution (see [9]) of

$$
\left\{\begin{array}{lr}
r(h x) \frac{\partial u}{\partial t}-\operatorname{div}(a(h x, t) \cdot D u)=f & \text { in } \Omega \times(0, T) \\
u=0 & \text { in } \partial \Omega \times(0, T) \\
u=\varphi & \text { in } \Omega_{h,+} \times\{0\}
\end{array}\right.
$$

converge in $L^{2}\left(0, T ; L^{2}(\Omega)\right)$ to the solution of the problem

$$
\left\{\begin{array}{lr}
{\left[\frac{1}{|Q|} \int_{Q} r\right] \frac{\partial u}{\partial t}-\sum_{i, j=1}^{n} \widehat{a}_{i j}(t) \frac{\partial^{2} u}{\partial x_{i} \partial x_{j}}=f} & \text { in } \Omega \times(0, T) \\
u=0 & \text { in } \partial \Omega \times(0, T) \\
u=\varphi & \text { in } \Omega \times\{0\}
\end{array}\right.
$$

(for the definition of $\widehat{a}$ see for example [9]). Notice that if the mean value of $r|Q|^{-1} \int_{Q} r>0$ the limit problem is given by a standard parabolic equation and in this case the initial condition is obtained in all $\Omega$ even if for every $h$ the problems above are partially elliptic and partially parabolic. This happens also if $r$ is positive only on a Cantor set of positive measure.

The only case in which the limit problem is elliptic is when $r \equiv 0$ : in this case problems (46) are the sequence of elliptic problems

$$
\left\{\begin{array}{lr}
-\operatorname{div}(a(h x, t) \cdot D u)=f(x, t) & \text { in } \Omega \\
u=0 & \text { in } \partial \Omega
\end{array} \quad \text { for a.e. } t \in(0, T)\right.
$$

and the initial conditions $u=\varphi$ in $\Omega_{h,+} \times\{0\}$ are meaningless. 


\subsection{Singular perturbations}

8) Suppose to have a fixed elliptic operator $u \mapsto-\operatorname{div}(a(x, t) \cdot D u)$, with $a \in \mathcal{M}_{\Omega \times(0, T)}\left(\lambda_{0}, \Lambda_{0}, M\right)$. Then $a \stackrel{G}{\longrightarrow} a$ in particular means that the solutions of

$$
\left\{\begin{array}{lr}
\frac{1}{h} \frac{\partial u}{\partial t}-\operatorname{div}(a \cdot D u)=f & \text { in } \Omega \times(0, T) \\
u=0 & \text { in } \partial \Omega \times(0, T) \\
u=\varphi & \text { in } \Omega \times\{0\}
\end{array}\right.
$$

converge in $L^{2}\left(0, T ; L^{2}(\Omega)\right)$ to the solution of the problem

$$
\left\{\begin{array}{lr}
-\operatorname{div}(a \cdot D u)=f & \text { in } \Omega \times(0, T) \\
u=0 & \text { in } \partial \Omega \times(0, T) .
\end{array}\right.
$$

Indeed $r_{h}=1 / h$ and $r \equiv 0$ belong to the class $\mathcal{F}\left(C_{1}, C_{2}, \mu_{0}\right)$.

But in fact we have more. The following convergence result holds.

Proposition 5.1. Consider $\mathcal{A}, \mathcal{A}_{h}: L^{2}\left(0, T ; H_{0}^{1}(\Omega)\right) \rightarrow L^{2}\left(0, T ; H^{-1}(\Omega)\right)$ the operators $\mathcal{A} u=-\operatorname{div}(a(x, t)$. $D u), \mathcal{A}_{h} u=-\operatorname{div}\left(a_{h}(x, t) \cdot D u\right)$ with $a, a_{1}, a_{2}, \ldots \in \mathcal{M}_{\Omega \times(0, T)}\left(\lambda_{0}, \Lambda_{0}, M, N\right)$. Suppose $\mathcal{A}_{h} v \rightarrow \mathcal{A} v$ in $L^{2}\left(0, T ; H^{-1}(\Omega)\right)$ for every $v \in L^{2}\left(0, T ; H_{0}^{1}(\Omega)\right)$. Then

$$
\begin{array}{ll}
u_{h} \rightarrow u & \text { in } L^{2}\left(0, T, H_{0}^{1}(\Omega)\right) \\
a_{h} \cdot D u_{h} \rightarrow a \cdot D u & \text { in } L^{2}\left(0, T, L^{2}(\Omega)^{n}\right),
\end{array}
$$

where $u_{h}$ and $u$ are respectively the solutions to (18) and (29), and $a_{h} \stackrel{G}{\longrightarrow} a$.

Proof. Since $\mathcal{A}_{h}-\mathcal{A}=\mathcal{A}_{h}\left(\mathcal{A}^{-1}-\mathcal{A}_{h}^{-1}\right) \mathcal{A}$, fix $f \in L^{2}\left(0, T ; H^{-1}(\Omega)\right)$ and choose $v=\mathcal{A}^{-1} f$. Then

$$
\mathcal{A}^{-1} f-\mathcal{A}_{h}^{-1} f=\mathcal{A}_{h}^{-1}\left(\mathcal{A}_{h} v-\mathcal{A} v\right)
$$

By our assumptions and (16) we conclude that $\left\|\mathcal{A}_{h}^{-1} f-\mathcal{A}^{-1} f\right\|_{L^{2}\left(0, T, H_{0}^{1}(\Omega)\right)} \rightarrow 0$. In particular $\| \mathcal{A}_{h}^{-1} f-$ $\mathcal{A}^{-1} f \|_{L^{2}\left(0, T, L^{2}(\Omega)\right)} \rightarrow 0$ and, for every $\Phi \in L^{2}\left(0, T ; L^{2}(\Omega)^{n}\right)$ by $(12)$

$$
\begin{aligned}
\sup _{\|\Phi\|=1} \mid & \int_{0}^{T} \int_{\Omega}\left(a_{h} \cdot D \mathcal{A}_{h}^{-1} f-a \cdot D \mathcal{A}^{-1} f, \Phi\right) \mathrm{d} x \mathrm{~d} t \mid \\
& \leqslant c\left(M, \Lambda_{0}\right)\left\langle\mathcal{A}_{h} u-\mathcal{A} u, u\right\rangle_{L^{2}\left(0, T ; H^{-1}(\Omega)\right) \times L^{2}\left(0, T ; H_{0}^{1}(\Omega)\right)} \rightarrow 0 .
\end{aligned}
$$

By Theorem 4.5 we derive that $a_{h} \stackrel{G}{\longrightarrow} a$.

Remark 5.2. Observe that assumptions of Proposition 5.1 are guaranteed if $\left(a_{h}\right)_{h} \subset \mathcal{M}_{\Omega \times(0, T)}\left(\lambda_{0}, \Lambda_{0}, M, N\right)$ and $a_{h} \rightarrow a$ in $L_{l o c}^{1}(\Omega \times(0, T))$, but the converse is not true (see [14]).

We conclude that under assumptions of Proposition 5.1, for every $\left(r_{h}\right)_{h} \subset \mathcal{F}\left(C_{1}, C_{2}, \mu_{0}\right)$ converging to $r \in \mathcal{F}\left(C_{1}, C_{2}, \mu_{0}\right)$ in $L^{\infty}(\Omega \times(0, T))$-weak*, we have that

$$
\begin{array}{ll}
u_{h} \rightarrow u & \text { in } L^{2}\left(0, T, H_{0}^{1}(\Omega)\right) \\
a_{h} \cdot D u_{h} \rightarrow a \cdot D u & \text { in } L^{2}\left(0, T, L^{2}(\Omega)^{n}\right),
\end{array}
$$


where $u_{h}$ and $u$ denote respectively the solutions of

$$
\left\{\begin{array} { l r } 
{ \partial _ { t } ( r _ { h } v ) - \operatorname { d i v } ( a _ { h } \cdot D v ) = f \Omega \times ( 0 , T ) } \\
{ v = 0 } \\
{ v = \varphi } \\
{ \Omega _ { h , + } ( 0 ) \times \{ 0 \} }
\end{array} \quad \left\{\begin{array}{lr}
\partial_{t}(r v)-\operatorname{div}(a \cdot D v)=f \Omega \times(0, T) \\
v=0 \\
v=\varphi & \partial \Omega \times(0, T) \\
\Omega_{+}^{r}(0) \times\{0\} .
\end{array}\right.\right.
$$

Acknowledgements. The author wish to thank the anonymous Referee for the many precious suggestions.

\section{REFERENCES}

[1] R.W. Carroll and R.E. Showalter, Singular and Degenerate Cauchy Problems. Academic Press, New York (1976).

[2] V. Chiadò Piat, G. Dal Maso and A. Defranceschi, G-convergence of monotone operators. Ann. Inst. H. Poincaré, Anal. Non Linéaire 7 (1990) 123-160.

[3] F. Colombini and S. Spagnolo, Sur la convergence de solutions d'équations paraboliques. J. Math. Pur. Appl. 56 (1977) $263-306$.

[4] G. Dal Maso, An introduction to $\Gamma$-convergence. Birkhäuser, Boston (1993).

[5] E. De Giorgi and S. Spagnolo, Sulla convergenza degli integrali dell'energia per operatori ellittici del secondo ordine. Boll. Un. Mat. Ital. 8 (1973) 391-411.

[6] L.C. Evans and R.F. Gariepy, Measure Theory and Fine Properties of Functions. CRC Press, USA (1992).

[7] A. Pankov, G-convergence and Homogenization of Nonlinear Partial Differential Operators. Kluwer Academic Publishers, Dordrecht (1997).

[8] F. Paronetto, Existence results for a class of evolution equations of mixed type. J. Funct. Anal. 212 (2004) $324-356$.

[9] F. Paronetto, Homogenization of degenerate elliptic-parabolic equations. Asymptotic Anal. 37 (2004) 21-56.

[10] R.E. Showalter, Degenerate parabolic initial-boundary value problems. J. Diff. Eq. 31 (1979) 296-312.

[11] R.E. Showalter, Monotone Operators in Banach Space and Nonlinear Partial Differential Equations. American Mathematical Society (1997).

[12] J. Simon, Compact sets in the space $L^{p}(0, T ; B)$. Ann. Mat. Pura Appl. 146 (1987) 65-96.

[13] S. Spagnolo, Sul limite delle soluzioni di problemi di Cauchy relativi all'equazione del calore. Ann. Scuola Norm. Sup. Pisa Cl. Sci. 21 (1967) 657-699.

[14] S. Spagnolo, Sulla convergenza di soluzioni di equazioni paraboliche ed ellittiche. Ann. Scuola Norm. Sup. Pisa Cl. Sci. 22 (1968) 571-597.

[15] S. Spagnolo, Convergence of parabolic equations. Boll. Un. Mat. Ital. 14-B (1977) 547-568.

[16] L. Tartar, Convergence d'operateurs defferentiels, Proceedings of the Meeting "Analisi convessa e Applicazioni". Roma (1974).

[17] L. Tartar, Cours Peccot, Collège de France, 1977. Partially written in: F. Murat, H-convergence - Séminaire d'Analyse Fonctionnelle et Numérique, Université d'Alger, 1977-78. English translation: F. Murat and L. Tartar: H-Convergence, in Topics in the Mathematical Modelling of Composite Materials, A. Cherkaev, R. Kohn, Editors, Birkhäuser, Boston (1997) $21-43$. 